\title{
Optimization of the Stirring Blade Structure of the Pumping Unit Based on the Improvement of Concrete Suction Efficiency
}

\author{
Shengqiang Jiang $\mathbb{D}^{1},{ }^{1}$ Hong Wan, ${ }^{1}$ Guodong Cao ${ }^{D},{ }^{1}$ Yuanqiang Tan, ${ }^{2}$ Jingang Liu, \\ Shiping Yang, ${ }^{1}$ Xiangwu Xiao, ${ }^{1}$ Zhenggang Tong, ${ }^{3}$ and Quanxu Yu ${ }^{3}$ \\ ${ }^{1}$ School of Mechanical Engineering, Xiangtan University, Xiangtan 411105, China
${ }^{2}$ Institute of Manufacturing Engineering, Huaqiao University, Xiamen 361021, China
${ }^{3}$ SANY Industial Park, SANY Heavy Industry Co., Ltd.,, Changsha 411100, China
}

Correspondence should be addressed to Shengqiang Jiang; jsqcx@xtu.edu.cn

Received 3 December 2021; Accepted 27 January 2022; Published 25 February 2022

Academic Editor: Andreas Lampropoulos

Copyright (c) 2022 Shengqiang Jiang et al. This is an open access article distributed under the Creative Commons Attribution License, which permits unrestricted use, distribution, and reproduction in any medium, provided the original work is properly cited.

\begin{abstract}
In this paper, a discrete element method (DEM) is used to simulate the suction process of fresh concrete in the pumping system, and the influence of the stirring blade on the suction is explored. The Hertz-Mindlin with JKR Cohesion contact model is used to establish the DEM model of fresh concrete, and the suction work is realized by an API function of DEM, and the movement of particles in the suction cylinder can be completed smoothly. The changes of the concrete flow field during the suction process are further studied, and the influence mechanism of the stirring blade on the suction process is explained. A numerical simulation scheme is designed to explore the influence of the rotation speed, installation angle, and edges distance of the stirring blade on the suction efficiency and the stirring resistance torque, and the structure of the stirring blade is optimized according to the influence law. The simulation results show that the stirring resistance torque of the optimized stirring blade is reduced without the suction efficiency of the pumping system reduced, and the stirring energy consumption of the pump suction is reduced.
\end{abstract}

\section{Introduction}

Fresh concrete is transported from the hopper to the pouring site along the pipeline with the help of the concrete pump truck [1]. It has the advantages of adjustable pumping height, long conveying distance, large pumping concrete quality, fast conveying speed, high efficiency, continuous operation, labor saving, and strong economy. And it is widely used in various concrete construction projects [2]. Pumping system is the core part of the concrete pump truck [3], usually located in its tail. The reciprocating motion of the piston in the conveying cylinder is powered by the hydraulic system that converts the hydraulic energy into mechanical energy. With the cooperation of the S-pipe reversing work, the concrete in the hopper is sucked into the conveying cylinder to complete the suction work, while the concrete in the conveying cylinder is pushed through the S-pipe to the output pipe to complete the push work, as shown in Figure 1. During the pumping process, the stirring blade continuously stirs the fresh concrete in the hopper. Speeding up the flow of the fresh concrete that can ensure the fluidity of the fresh concrete in the hopper, promote the suction work, prevent the rapid solidification of the fresh concrete, and the blockage of the pipe at the bottom of the hopper. Optimizing the structural parameters of the stirring blade can improve the suction performance and reduce the energy consumption.

The actual experiments of pumping concrete are timeconsuming and labor-intensive, while the numerical simulation technology can reduce the waste of resources and save the economy. In recent years, there were many numerical simulations of fresh concrete. Roussel et al. [4] roughly divided its types into three types: single-phase flow models [5-7], discrete particle flow models [8-10], and suspension flow models [11-13] and elaborated on the advantages and disadvantages of the three methods. Choi et al. [14] chose the single-phase fluid method and used 


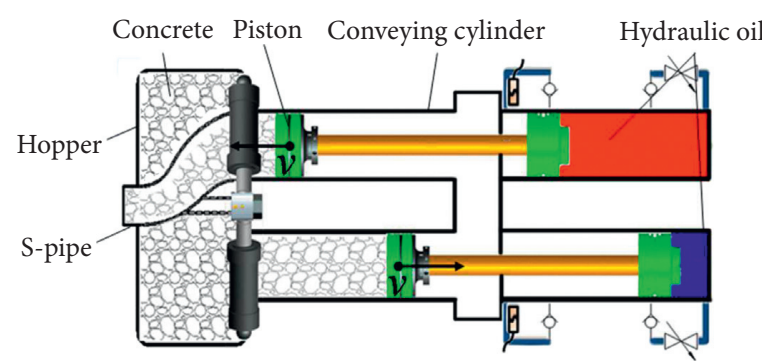

(a)

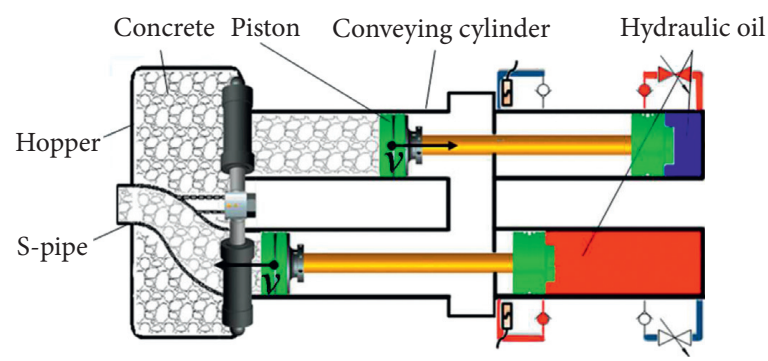

(b)

Figure 1: The pumping process before (a) and after (b) the S-pipe reversing work.

Fluent to solve the complex concrete flow. The experimental, analytical, and numerical results found that the behavior of the lubricating layer is similar to that of mortar in terms of rheology. Zhan et al. [15] used the DEM to numerically simulate the local pumping problem and explored the influence of a series of factors on the local pumping performance, which were related to the pipe geometry, aggregate geometry, and pumping conditions. Shengqiang et al. [16] used a computational fluid dynamics-discrete element method (CFD-DEM) coupling model to explore the influence of structural parameters and inclination angles of tapered pipes, elbows and their combinations on pressure loss, and proposed a pump truck conveying pipeline. They used a single-objective optimization method to reduce the pressure loss (about 5.45\%) in the pumping process. In addition, there were a lot of work on the optimal design of pumps [17-19]. Liu et al. [20] explored the influence of the geometry of inlet guide vanes (IGVs) on the stability of centrifugal pump operation, and prerotation adjustment of IGVs effectively adjusted the operation of centrifugal pump. The researches on pumping were mostly aimed at the pumping and flow of fresh concrete in the pipeline, but the researches on the process of concrete pumping and suction are very few. At present, there is still a lack of research on the suction process of the pumping system.

In this paper, the discrete element method simulated the suction process of fresh concrete in the pumping system, and the influence of the stirring blade on the suction is explored. First, the Hertz-Mindlin with JKR Cohesion contact model is used to establish the DEM model of fresh concrete, and the mechanical behavior of the concrete particles is described. Comparison between experiments and simulations of slump flow tests and L-box tests, the feasibility of simulating the flow behavior and rheological properties of the fresh concrete is proved. The geometric structure of pumping system is simplified. The suction force of the conveying cylinder is customized by the API function of DEM to realize the suction work; therefore, the numerical simulation of the suction process is completed. Next, the changes of the concrete flow field during the suction process are further studied, the particle flow velocity at different positions is considered, and the influence mechanism of the stirring blade on suction process is explained. In addition, the numerical simulation scheme is designed to explore the influence of the rotation speed, installation angle and edges distance of the stirring blade on the suction efficiency and the stirring resistance torque, and the structure of the stirring blade is optimized according to the influence law. Finally, verified by the results before and after optimization, the stirring resistance torque of the optimized stirring blade is reduced without the suction efficiency of the pumping system reduced, thereby the stirring energy consumption of the pump suction is reduced.

\section{The Simulation of the Suction Process of the Pumping System}

2.1. The Numerical Simulation Method. The discrete element method is that the discontinuous body is separated into a set of rigid elements, and the motion equation of each rigid element is solved by the method of time-step iteration, and then the overall motion shape of the discontinuous body is obtained. In the process of numerical simulation using the discrete element method, each particle in the material is used as a particle unit to establish a mathematical model, and the size and physical properties of the particle unit are given. Among them, there are two relations of contact and separation between each particle. When the contact occurs, the contact force and moment will be generated at the contact point, the magnitude of that can be calculated according to the contact mechanics model.

Hertz-Mindlin with JKR (Johnson-Kendall-Roberts) cohesion is a cohesion contact model that can model strongly adhesive systems and provide attractive cohesion forces. JKR normal force $F_{J K R}$, normal damping $F_{n}^{d}$, tangential force $F_{t}$, tangential damping $F_{t}^{d}$, and rolling frictional torque $T_{i}$ are as follows:

$$
\begin{aligned}
& F_{J K R}=-4 \sqrt{\pi \gamma E^{*}} \alpha^{(3 / 2)}+\frac{4 E^{*}}{3 R^{*}} \alpha^{3}, \\
& F_{n}^{d}=-2 \sqrt{\frac{5}{6}} \frac{\ln e}{\sqrt{\ln ^{2} e+\pi^{2}}} \sqrt{K_{n} m^{*}} V_{n}^{\text {rel }} \\
& F_{t}=-K_{t} \delta_{t} \\
& F_{t}^{d}=-2 \sqrt{\frac{5}{6}} \frac{\ln e}{\sqrt{\ln ^{2} e+\pi^{2}}} \sqrt{K_{n} m^{*}} V_{t}^{\mathrm{rel}} \\
& T_{i}=-\mu_{r} F_{J K R} R_{i} \omega_{i} .
\end{aligned}
$$


In the above,

$$
\begin{aligned}
& \delta_{n}=\frac{\alpha^{2}}{R^{*}}-\sqrt{\frac{4 \pi \alpha}{E^{*}}}, \\
& \frac{1}{E^{*}}=\frac{1-v_{i}^{2}}{E_{i}}-\frac{1-v_{j}^{2}}{E_{j}}, \\
& \frac{1}{R^{*}}=\frac{1}{R_{i}}+\frac{1}{R_{j}}, \\
& \frac{1}{m^{*}}=\frac{1}{m_{i}}+\frac{1}{m_{j}}, \\
& K_{n}=2 E^{*} \sqrt{R^{*} \delta_{n}}, \\
& K_{t}=8 G^{*} \sqrt{R^{*} \delta_{n}} .
\end{aligned}
$$

Figure 2 shows the typical plot of JKR normal force as a function of normal overlap.

This model provides attractive cohesion forces even if the particles are not in physical contact. The maximum gap between particles with nonzero force is given by

$$
\begin{aligned}
& \delta_{C}=\frac{\alpha_{C}^{2}}{R^{*}}-\sqrt{\frac{4 \pi \gamma \alpha_{C}}{E^{*}}} \\
& \alpha_{C}=\left[\frac{9 \pi \gamma R^{* 2}}{2 E^{*}}\left(\frac{3}{4}-\frac{1}{\sqrt{2}}\right)\right]^{(1 / 3)} .
\end{aligned}
$$

The maximum value of the cohesion force occurs when particles are not in physical contact and the separation gap is less than $\delta_{c}$. The value of maximum cohesion force, called pull-out force, is given by

$$
F_{J \text { pullout }}=-\frac{3}{2} \pi \gamma R^{*}
$$

Hence, the adhesion of fresh concrete is determined by the surface energy, which is the basic parameter of the Hertz-Mindlin with JKR model. The viscosity of concrete flow is characterized by the adhesion of fresh concrete.

2.2. The DEM Model of Fresh Concrete. Fresh concrete is mainly mixed by water, cement, sand, coarse aggregate (stone), and various additives (concrete water-reducing agent or concrete retarder). The mix proportions for the fresh concrete are given in Table 1 . The mass ratio of mortar (water + cement + sand + water-reducing agent + retarder) to coarse-aggregate is about 1.35 .

In the simulation, the fresh concrete is composed of coarse aggregate particles and mortar particles with a mass ratio of 1.35 . In order to realize the feasibility of numerical simulation and the calculation efficiency, both particles are set as spheres. The diameter of mortar particles is uniformly $10 \mathrm{~mm}$. According to experiments, the coarse aggregate particle size in the DEM model is normally distributed, with an average of $20 \mathrm{~mm}$, a standard deviation of $5 \mathrm{~mm}$, a

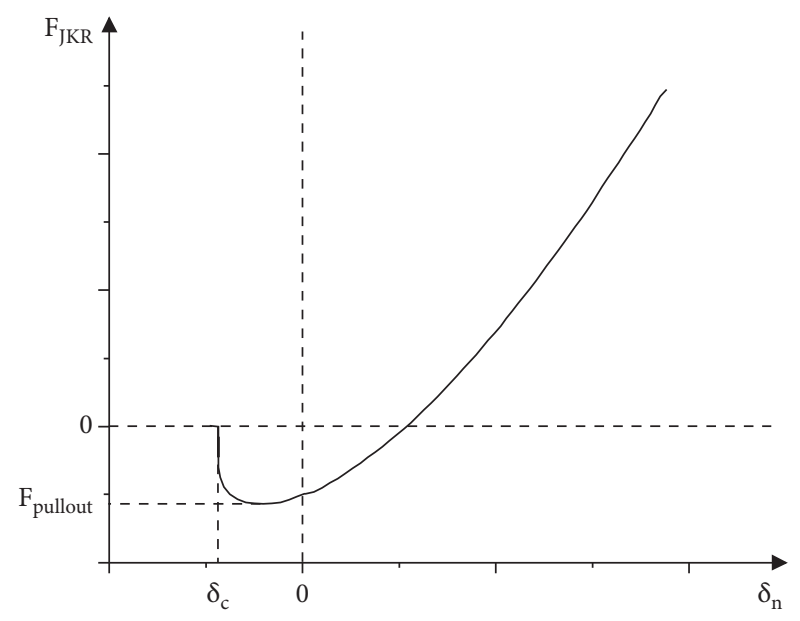

Figure 2: Normal force as a function of normal overlap.

minimum of $10 \mathrm{~mm}$, and a maximum of $30 \mathrm{~mm}$, as shown in Figure 3.

Under the hydration of cement, fresh concrete has strong adhesion. The adhesion not only exists between particles and particles but also particles and geometry. Compared with other contact models, the Hertz-Mindlin with JKR model has higher matching for fresh concrete, which has obvious cohesion effects [21]. The mechanical behaviors of fresh concrete discrete elements are described when they come into contact with each other.

2.3. The Validation of the Fresh Concrete DEM Model. The rheological properties of fresh concrete are expressed by various standard tests, slump flow test, L-box test, and V-funnel test. In experiments, three slump tests and L-box tests were repeated, and the test results are averaged. In the simulations of slump flow tests and L-box tests, all the uncertain quantities in the DEM model of the fresh concrete were continuously adjusted so that the gap between experiments and simulations results is less than $5 \%$. Figures 4(a) and 4(b) show the model of slump cone and L-box. Figures 4(c) and 4(d) show their dimensions.

In experiments, after the fresh concrete collapses and stabilizes naturally, the average remaining height is $77 \mathrm{~mm}$, the average slump value is $223 \mathrm{~mm}$, and the average expansion value is $404 \mathrm{~mm}$. In the simulation, the average remaining height is $85 \mathrm{~mm}$, the slump value is $215 \mathrm{~mm}$, which is $3.6 \%$ different from the experiments, and the expansion value is $413 \mathrm{~mm}$, which is $2.2 \%$ different from the experiments. It is not difficult to find that the simulation is very similar to the experiments by observing the vertical and horizontal shape of fresh concrete carefully from Figures 5(a) and 5(b).

In experiments, when the fresh concrete stopped flowing naturally, the fresh concrete did not flow to the end of the L-box. The average height of the front end is $219 \mathrm{~mm}$, and the flow distance is $480 \mathrm{~mm}$. In the simulation, the average height of the front end is $224 \mathrm{~mm}$, which is $2.3 \%$ different from the experiments, and the flow distance is $498 \mathrm{~mm}$, which is $3.8 \%$ different from the experiments. Figures 6(a) 
TABle 1: The mix proportions of the fresh concrete.

Water-cement ratio Cement $\left(\mathrm{kg} / \mathrm{m}^{3}\right)$ Sand $\left(\mathrm{kg} / \mathrm{m}^{3}\right)$ Coarse aggregate $\left(\mathrm{kg} / \mathrm{m}^{3}\right)$ Concrete water reducing $\left(\mathrm{kg} / \mathrm{m}^{3}\right)$ Concrete retarder

$\left(\mathrm{kg} / \mathrm{m}^{3}\right)$

\begin{tabular}{llllll}
\hline 0.42 & 390 & 900 & 1080 & 3.20 & 0.39
\end{tabular}

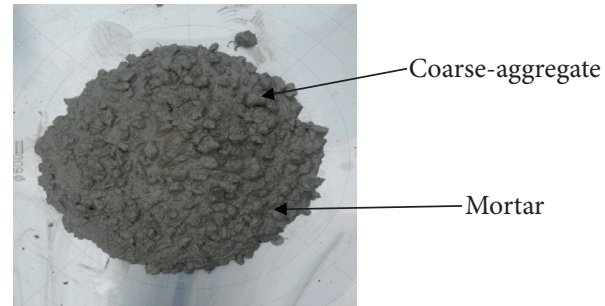

(a)

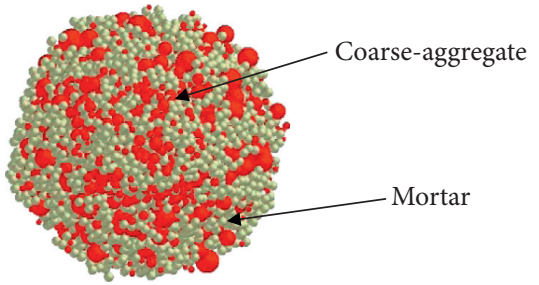

(b)

FIgURE 3: The fresh concrete in experiments (a) and simulations (b).
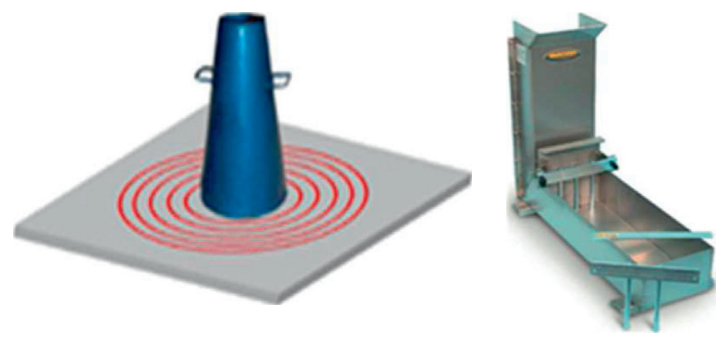

(a)

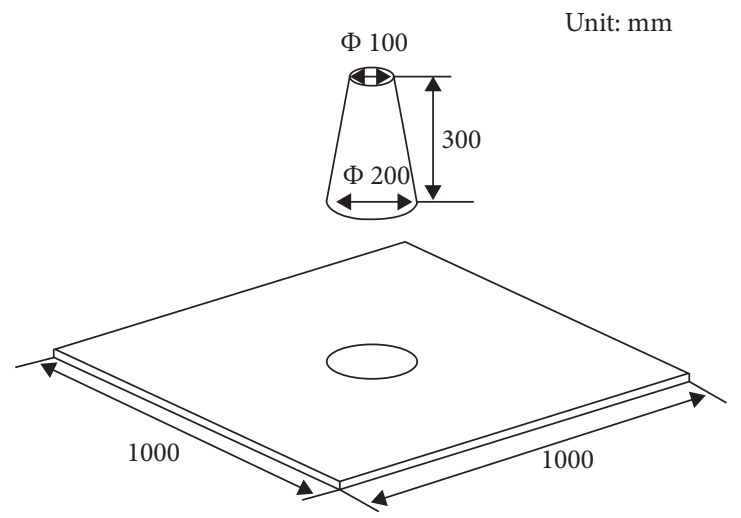

(c)

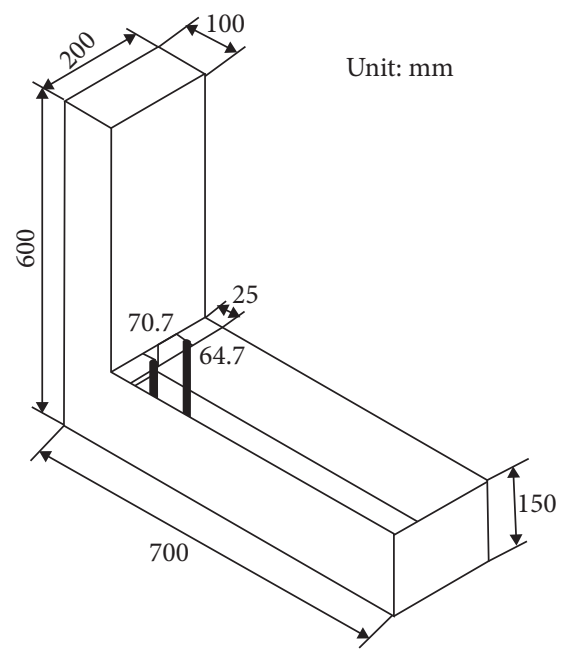

(d)

Figure 4: The model of the slump cone (a) and L-box (b); the dimension of the slump cone (c) and L-box (d).

and 6(b) show that the experiments and simulations of the L-box are also very similar.

In short, the difference between the results of simulations and experiments is less than $5 \%$ for slump and L-box tests. The feasibility of simulating the flow behavior and rheological properties of the fresh concrete is proved. The DEM model of the fresh concrete will be applied to the numerical simulation of the pumping and suction process. The surface energy of the final DEM model of fresh concrete is shown in Table 2. The time step is set as $3.80675 e-06 \mathrm{~s}$. 

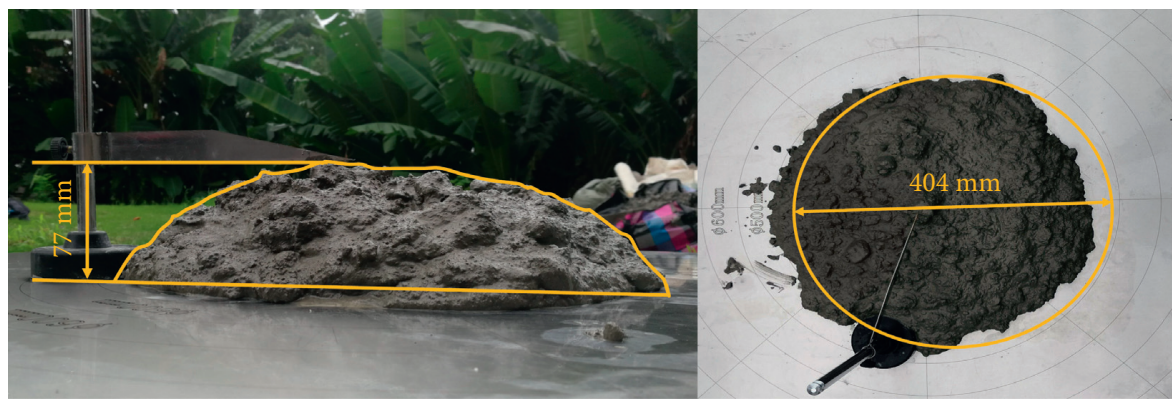

(a)
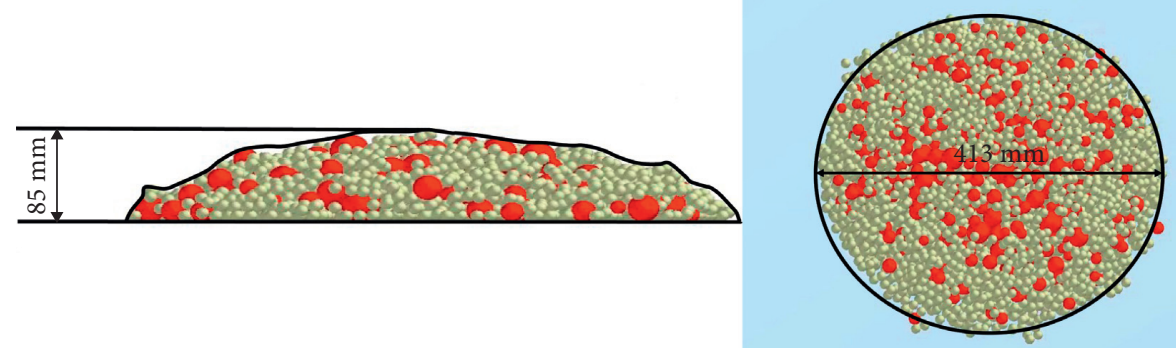

(b)

Figure 5: The results of slump tests in experiments (a) and simulations (b).

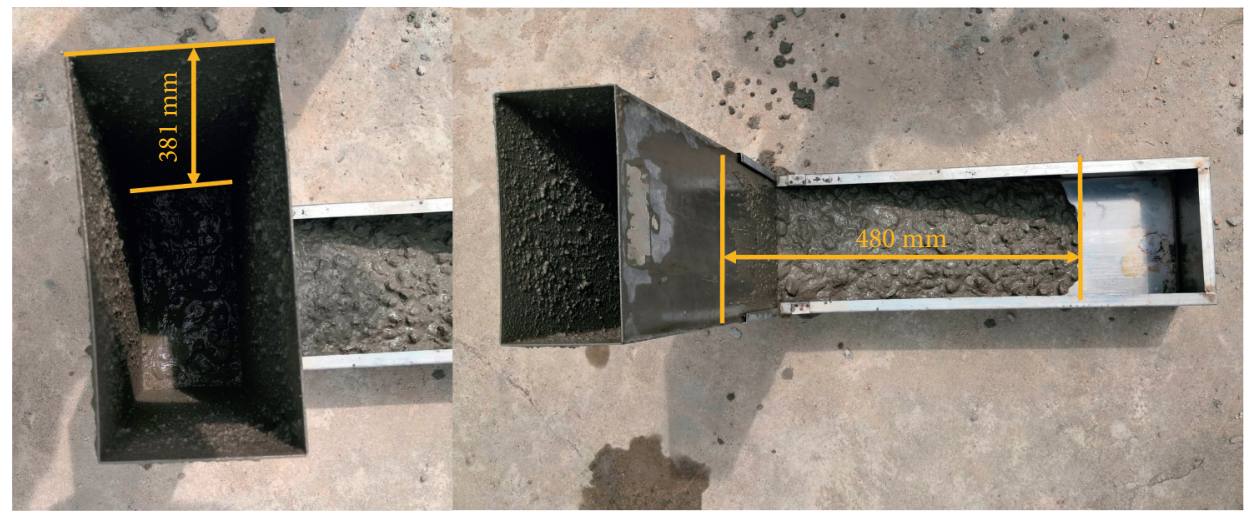

(a)

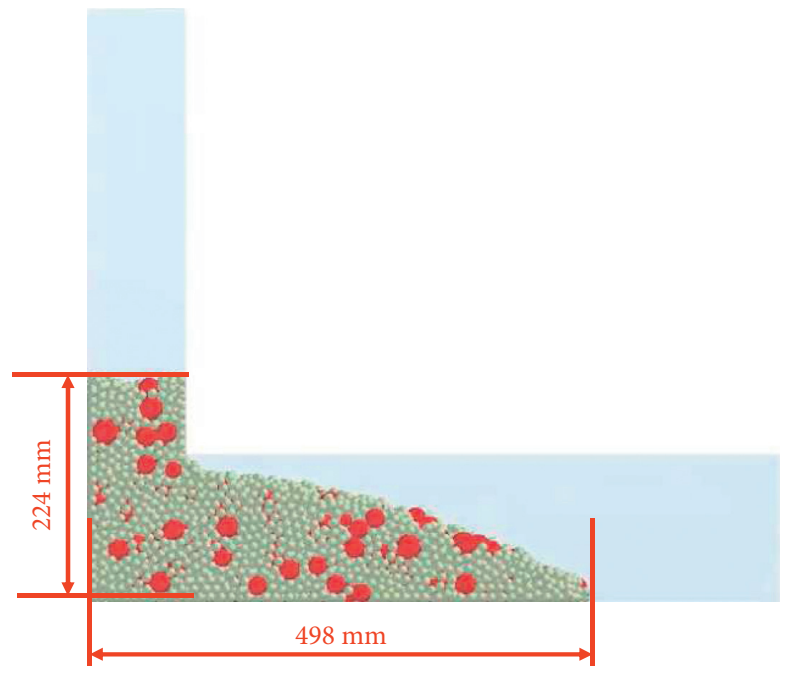

(b)

FIgURE 6: The results of L-box tests in experiments (a) and simulations (b). 
TABLe 2: The surface energy of the final DEM model of fresh concrete.

\begin{tabular}{|c|c|c|c|c|c|}
\hline & $\begin{array}{c}\text { Coarse aggregate-coarse } \\
\text { aggregate }\end{array}$ & $\begin{array}{c}\text { Coarse aggregate- } \\
\text { mortar }\end{array}$ & $\begin{array}{c}\text { Coarse aggregate- } \\
\text { steel }\end{array}$ & $\begin{array}{l}\text { Mortar- } \\
\text { mortar }\end{array}$ & Mortar-steel \\
\hline Surface energy $\left(\mathrm{J} / \mathrm{m}^{2}\right)$ & 1 & 3 & 0.6 & 5 & 0.9 \\
\hline
\end{tabular}

2.4. Realization of the Suction Work of the Pumping System. Pumping system is an executive mechanism to realize suction work and determine pumping performance, which is composed of pump transport mechanism, hopper, S valve, oscillating mechanism, stirring mechanism, conveying pipeline, and lubrication system. The pumping suction test device has an angle of $8^{\circ}$ with the horizontal plane, the inner diameter of the conveying cylinder is $230 \mathrm{~mm}$, and the pumping stroke is $1600 \mathrm{~mm}$, as shown in Figures $7(\mathrm{a})$ and 7(b).

In the DEM numerical simulation, the main purpose is to study the interaction between particles and particles and between particles and geometric bodies. Therefore, only the geometries in direct contact with the fresh concrete particles in the pumping system need to be considered when establishing geometric models in simulation. There is a hopper, a stirring blade, a S-pipe, two conveying cylinders $L$ and $R$, two concrete pistons $L$ and $R$, and an in-out pipe, as shown in Figure 8.

The suction efficiency is an important performance index of the pumping system, which is calculated as follows:

$$
\eta=\frac{M_{1}}{M_{2}}
$$

Among them, $M_{2}$ is theoretical suction mass that is the mass of the conveying cylinder completely filled with fresh concrete; $M_{1}$ is actual suction mass that is the mass of the conveying cylinder actually filled with fresh concrete during a single suction process.

The average suction efficiency is $84.6 \%$ that is measured in experiments. The theoretical suction mass is $88.9 \mathrm{~kg}$, which is measured in simulation.

In experiments, the hydraulic system converts hydraulic energy into mechanical energy, which drives the concrete piston to reciprocating motion in the conveying cylinder. The fresh concrete is sucked under the combined action of atmospheric pressure and gravity and pushed by the concrete piston. The suction process is mainly due to the movement of the concrete piston, and negative pressure is formed at the position of the piston port, which is manifested as the suction effect of the conveying piston cylinder.

In DEM numerical simulation, with the help of API function, an additional particle body force is defined as suction force. The concrete particles in the area near the connecting port of the conveying piston cylinder and the hopper obtain a custom suction force to realize the suction work at the beginning of each time step. As in experiments, the pushing process is realized by using concrete piston. The suction force is in the direction of the conveying cylinder in the suction process. Its size is proportional to the mass of the particle and the parameter $k$, which is defined to control the size of suction force. The relevant formulas are as follows:

$$
\begin{aligned}
F^{\prime} & =F+F_{\text {suction }}, \\
F_{\text {suction }} & =k m .
\end{aligned}
$$

In the above, $F^{\prime}$ is the resultant force of a particle at the beginning of the time step, $F$ is the resultant force of the particle at the end of the last time step, $F_{\text {suction }}$ is the selfdefined suction force, and $m$ is the mass of the particle.

Figure 9 (a) is the numerical simulation result without adding suction force $(k=0)$. The actual suction mass is $3.2 \mathrm{~kg}$, and the suction efficiency is $3.60 \%$. Figure $9(\mathrm{~b})$ is the numerical simulation result with adding suction force $(k=66)$. The actual suction mass is $75.2 \mathrm{~kg}$, and the suction efficiency is $84.59 \%$. The relationship between the parameter $k$ and suction efficiency is shown as Figure 10 according to the results of multiple numerical simulations. The value of parameter $k$ is adjusted to meet the experimental suction efficiency, which required adjustment for some conditions, including the inclination angle of the pumping system, the pumping stroke, and the speed of the piston movement during suction. In this article, the inclination angle of the pumping system is $8^{\circ}$, the pumping stroke is $1600 \mathrm{~mm}$, and the speed of the piston movement during suction is $1 \mathrm{~m} / \mathrm{s}$. Obviously, if suction force is not added, the actual suction mass is extremely small, and the suction work is difficult to complete. After adding suction force, the suction performance of the pumping system can be revealed. When $k$ is 66 , comparing the results in simulation with the results in experiments, the difference in suction efficiency is less than $0.1 \%$. Therefore, it is necessary to add suction force and $k$ is 66.

In the DEM numerical simulation, the total time is $7.7 \mathrm{~s}$. Fresh concrete particles were generated in the hopper from 0 to $0.5 \mathrm{~s}$. The movements of geometries are shown in Table 3 from 0.5 to $7.7 \mathrm{~s}$. The stirring blade rotates at a fixed speed. The S-pipe swings and commutates during the commutating period that is $0.2 \mathrm{~s}$. The stroke of the conveying cylinder is $1600 \mathrm{~mm}$, and the time for each suction and push is $1.6 \mathrm{~s}$. There is a pumping cycle of $3.6 \mathrm{~s}$, a total of 2 cycles. The numerical simulation visualization process of the second pumping cycle $(4.1-7.7 \mathrm{~s})$ is shown in Figure 11.

\section{Analysis of Concrete Flow}

3.1. The Flow Field of Concrete Particles. The particles velocity nephogram is used to represent the flow field of fresh concrete particles. In order to analyze the flow field in the pumping process, three sections $\mathrm{A}, \mathrm{B}$, and $\mathrm{C}$ are set up, as shown in Figure 12. Section $A$ is perpendicular to the central axis of $Z$ axis and passes through the central axis of the piston cylinder L. Section B is perpendicular to the central axis of $Y$ axis and passes through the central axis of the stirring blade. And section $C$ is perpendicular to the central 


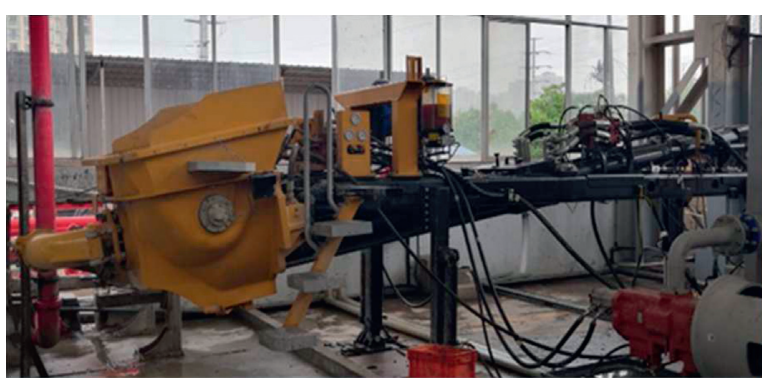

(a)

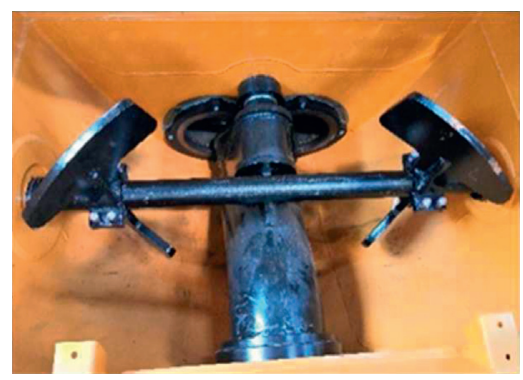

(b)

Figure 7: Pumping suction test device (a) and the internal situation of the hopper (b).

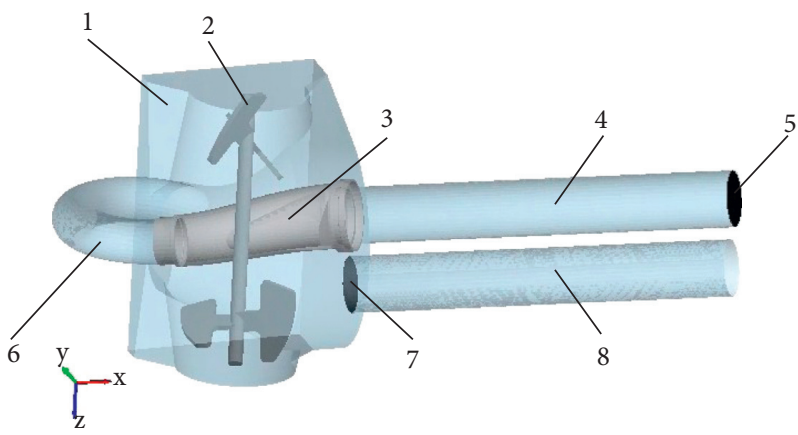
1-hopper, 2-stirring blade, 3-S-pipe, 4-conveying cylinder L,
5-concrete piston L, 6- in-out pipe, 7- concrete piston R, 8- conveying cylinder R.

Figure 8: The geometries in simulation. 1-hopper, 2-stirring blade, 3-S-pipe, 4-conveying cylinder $L$, 5-concrete piston $L$, 6-in-out pipe, 7 -concrete piston $R$, and 8-conveying cylinder $R$.

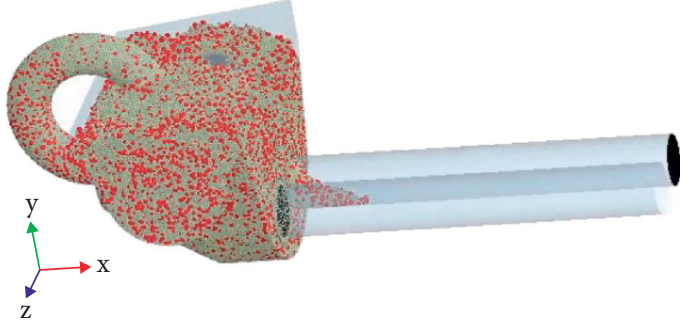

(a)

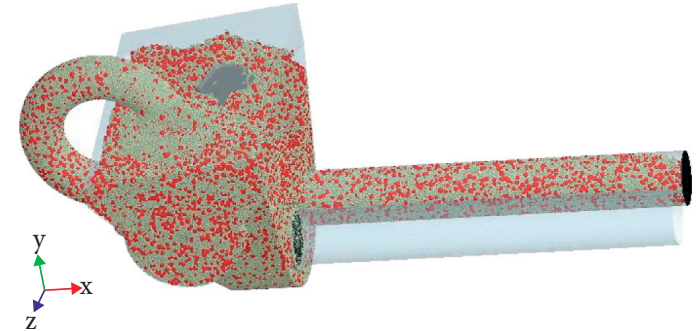

(b)

Figure 9: The numerical simulation results when $k=0$ (a) and $k=66$ (b).

axis of $X$ axis and passes through the central axis of the stirring blade.

At $5.9 \mathrm{~s}$, the S-pipe reversing is completed, and it is connected to the conveying cylinder $\mathrm{R}$. The concrete flow rate in the swing area of the S-pipe is larger, and the concrete flow rate near the suction side of the stirring area of the stirring blade is larger than that far from the suction side.

From 5.9 to $7.5 \mathrm{~s}$, the fresh concrete in the hopper is sucked into the conveying cylinder $L$, the $S$-pipe remains static, and the stirring blade keeps rotating. The concrete flow rate in the conveying cylinder $L$ is relatively stable in the early and middle stages and decreases at the end of the suction process. The concrete flow rate in the stirring area of stirring blade has little change. Figures 13(a)-13(d) show the concrete flow field of sections $\mathrm{A}, \mathrm{B}$, and $\mathrm{C}$ at $5.9 \mathrm{~s}, 6.4 \mathrm{~s}, 7 \mathrm{~s}$, and $7.5 \mathrm{~s}$.

From $7.5 \mathrm{~s}$ to $7.7 \mathrm{~s}$, the conveying cylinder $L$ stops suction, the S-pipe swings and reverses, and the stirring blade continues to rotate. The flow field in the hopper changes greatly, and the concrete flow rate away from the suction side increases affected by the reversal of the S-pipe. Figure 13(e) and 13(f) show the concrete flow field of sections $A, B$, and $C$ at $7.6 \mathrm{~s}$ and $7.7 \mathrm{~s}$.

According to the numerical simulation of the suction process, the concrete flow rate near the wall of hopper is small. The concrete flow rate in the stirring area of the 


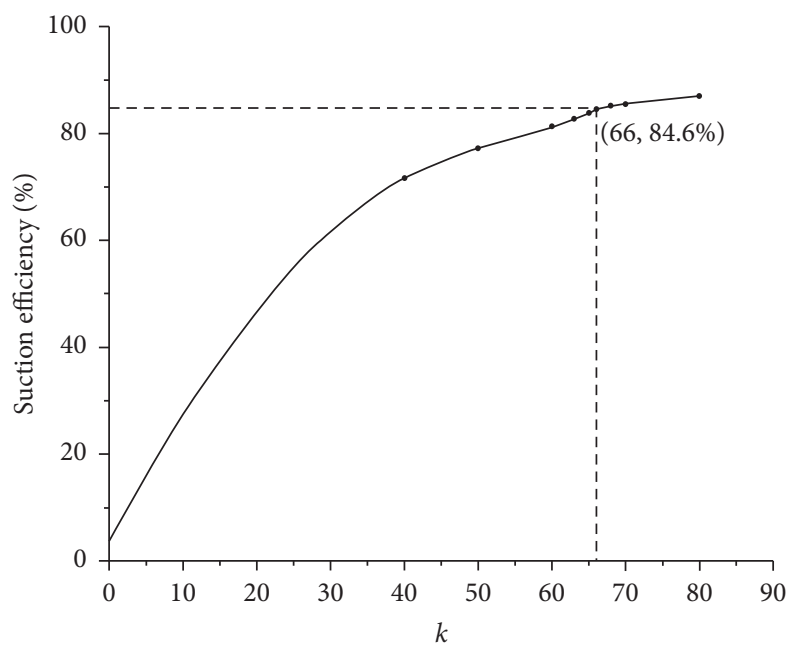

Figure 10: The relationship between the parameter $k$ and suction efficiency.

TABLE 3: The movements of geometries.

\begin{tabular}{lcccccc}
\hline Time & Stirring blade & S-pipe & Concrete piston $L$ & Conveying cylinder $L$ & Concrete piston $R$ & Conveying cylinder $R$ \\
\hline $0.5-2.1 \mathrm{~s}$ & & 0 & $-1 \mathrm{~m} / \mathrm{s}$ & Push & $1 \mathrm{~m} / \mathrm{s}$ & Suction \\
$4.1-5.9 \mathrm{~s}$ & & & & & & - \\
$2.1-2.3 \mathrm{~s}$ & & & & & 0 & Push \\
$5.7-5.9 \mathrm{~s}$ & $25 \mathrm{r} / \mathrm{min} / \mathrm{s}$ & 0 & $1 \mathrm{~m} / \mathrm{s}$ & Suction & $-1 \mathrm{~m} / \mathrm{s}$ & \\
$2.3-3.9 \mathrm{~s}$ & & 0 & & 0 & \\
$5.9-7.5 \mathrm{~s}$ & & $-6.12 \mathrm{rad} / \mathrm{s}$ & 0 & & & \\
$3.9-4.1 \mathrm{~s}$ & & &
\end{tabular}

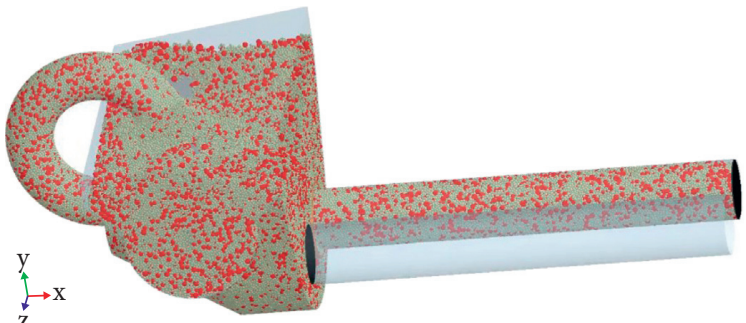

(a)

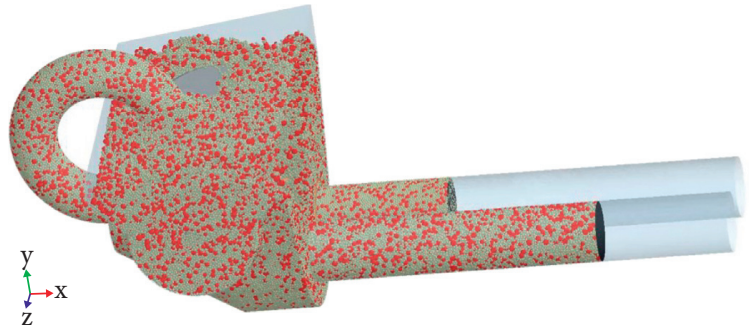

(c)

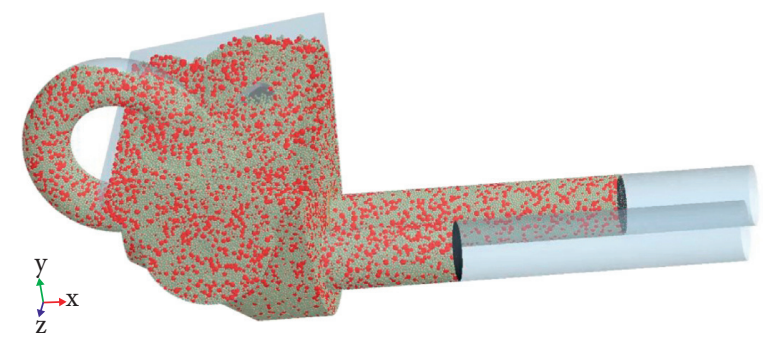

(b)

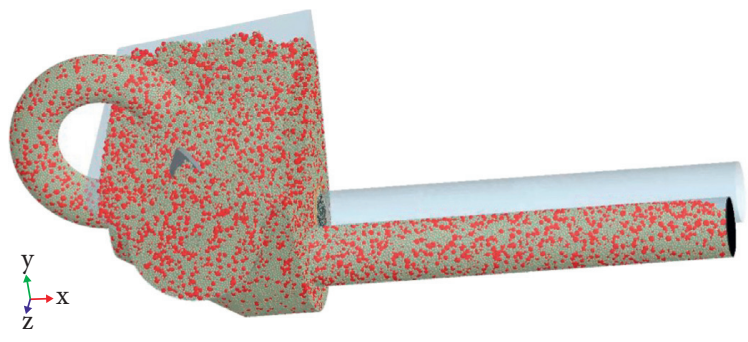

(d)

FIGURE 11: Continued. 


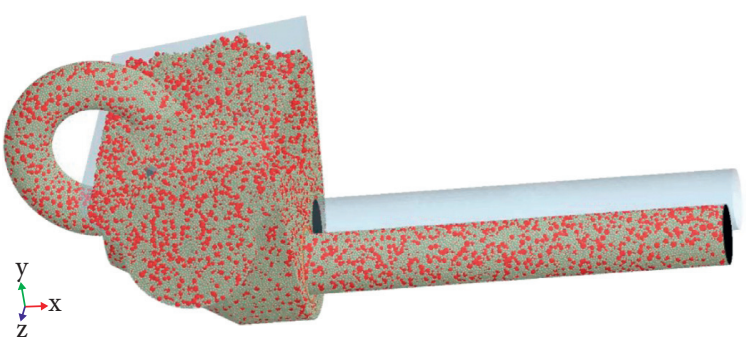

(e)

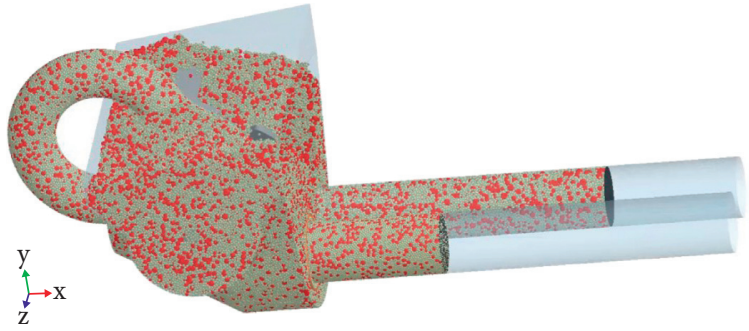

(g)

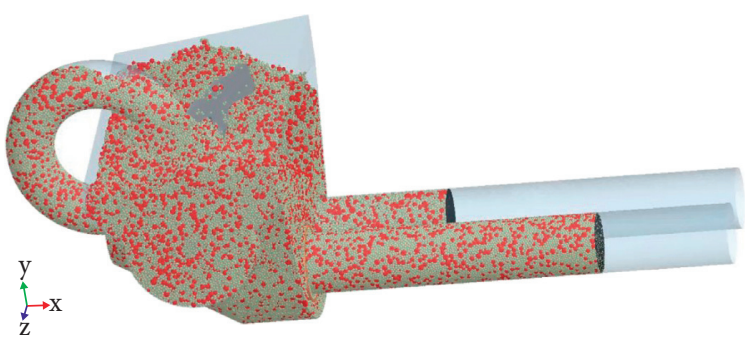

(f)

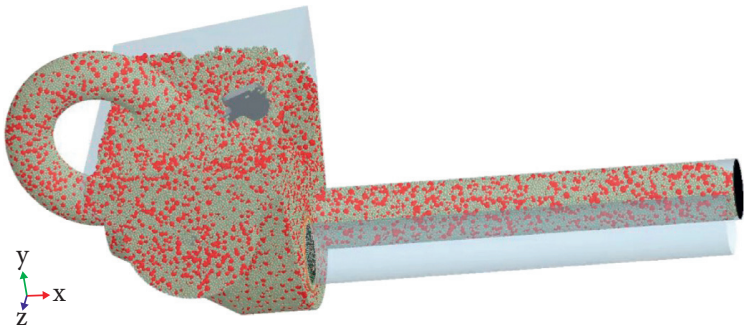

(h)

Figure 11: The numerical simulation visualization process of the second pumping cycle (4.1-7.7 s): (a) $t=4.1 \mathrm{~s}$, (b) $t=4.6 \mathrm{~s}$, (c) $t=5.2 \mathrm{~s}$, (d) $t=5.7 \mathrm{~s}$, (e) $t=5.9 \mathrm{~s}$, (f) $t=6.4 \mathrm{~s}$, (g) $t=7 \mathrm{~s}$, and (h) $t=7.56 \mathrm{~s}$.

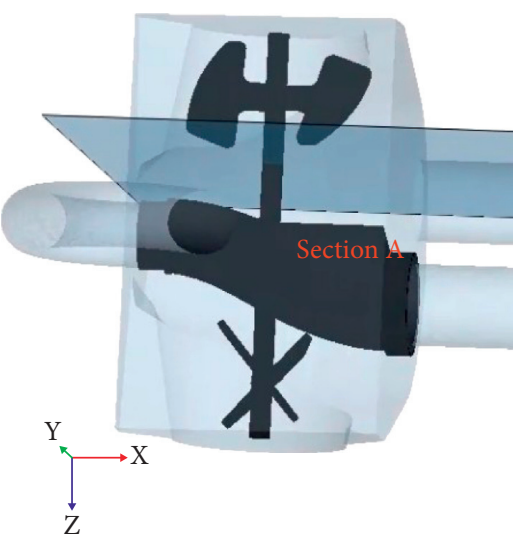

(a)

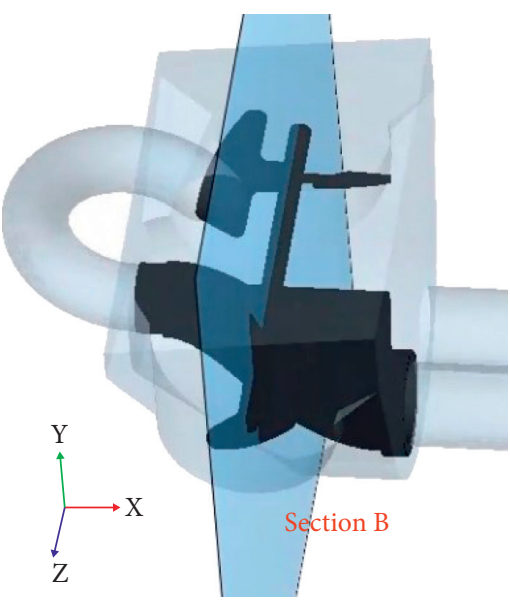

(b)

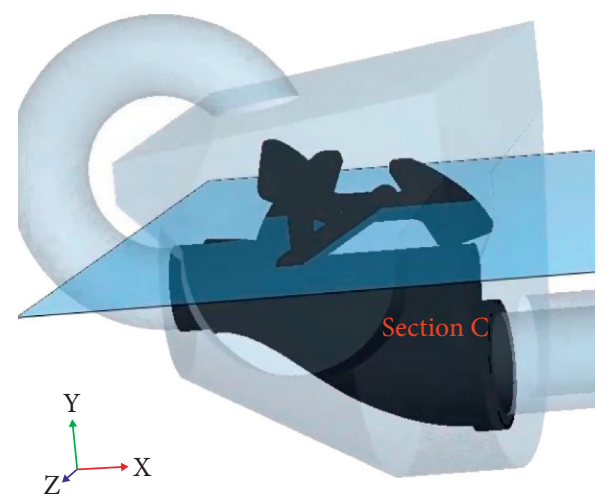

(c)

Figure 12: The position of sections.

stirring blade changes little. The concrete flow rate in the piston cylinder is large in the early and middle stages and is lower at the end of suction. When the S-pipe swings, the concrete flow rate in the swing area is large.

3.2. The Flow Velocity of Concrete Particles. There are 5 typical positions in the hopper, as shown in Figure 14. Position 1 is at the center of the connection between the conveying cylinder $L$ and the hopper, which is the necessary entrance and exit to suck and push fresh concrete. Position 2 is directly below the axis of the stirring blade, which is the edge of the rotation area of the stirring blade. Position 3 is located directly above the axis of the stirring blade and close to the shaft of the stirring blade, which is an area where the stirring blade cannot be swept. Position 4 is below the discharge end of the S-pipe, which is the area on the nonsuction side and close to the hopper wall in the hopper. Position 5 is located below the connection between the $\mathrm{S}$-pipe and the conveying cylinder $R$, which is the suction side and near the hopper wall area in the hopper. Each position has a virtual grid for counting the concrete particle velocity, which the size is $60 \mathrm{~mm} \times 60 \mathrm{~mm} \times 60 \mathrm{~mm}$. The center coordinates of grids are shown in Table 4.

Statistics of the concrete particle velocity at 5 locations, the change of particle velocity at different locations with time, and the average particle velocity are shown in Figure 15 . The change cycle of the particle velocity at position 1 

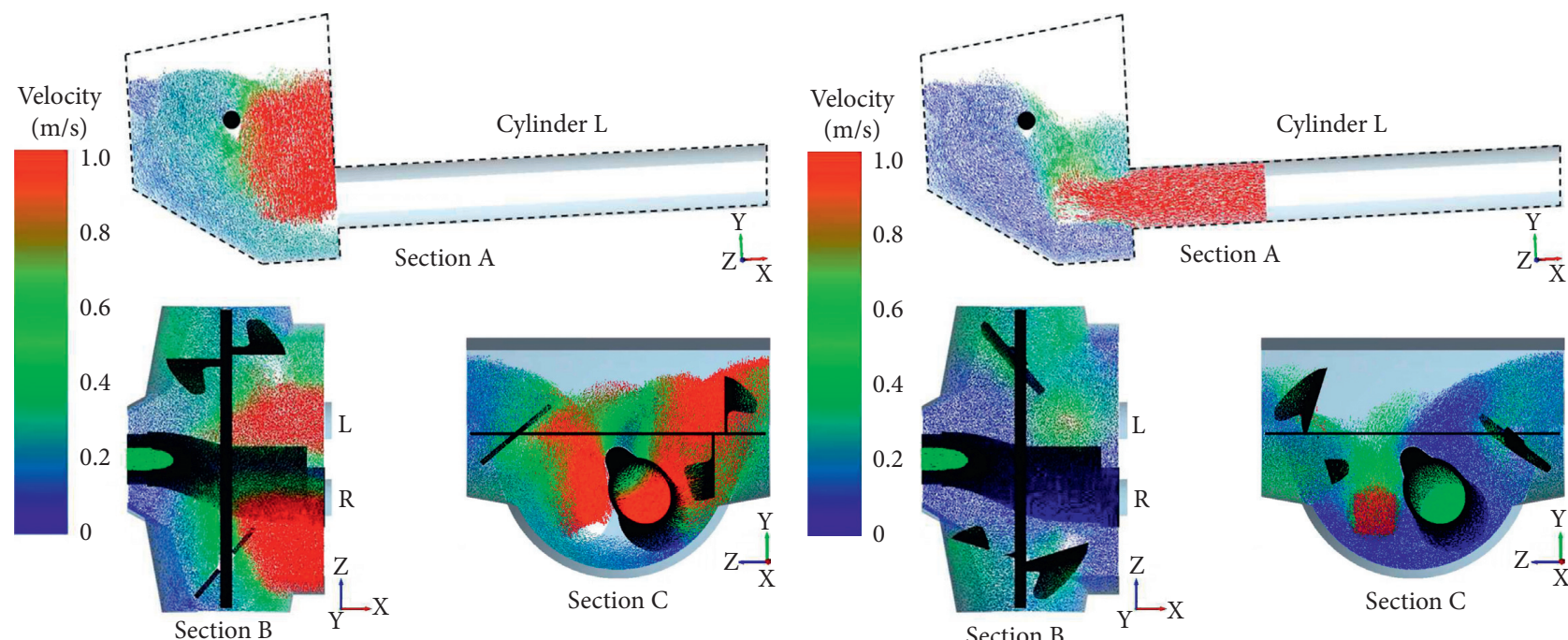

(a)
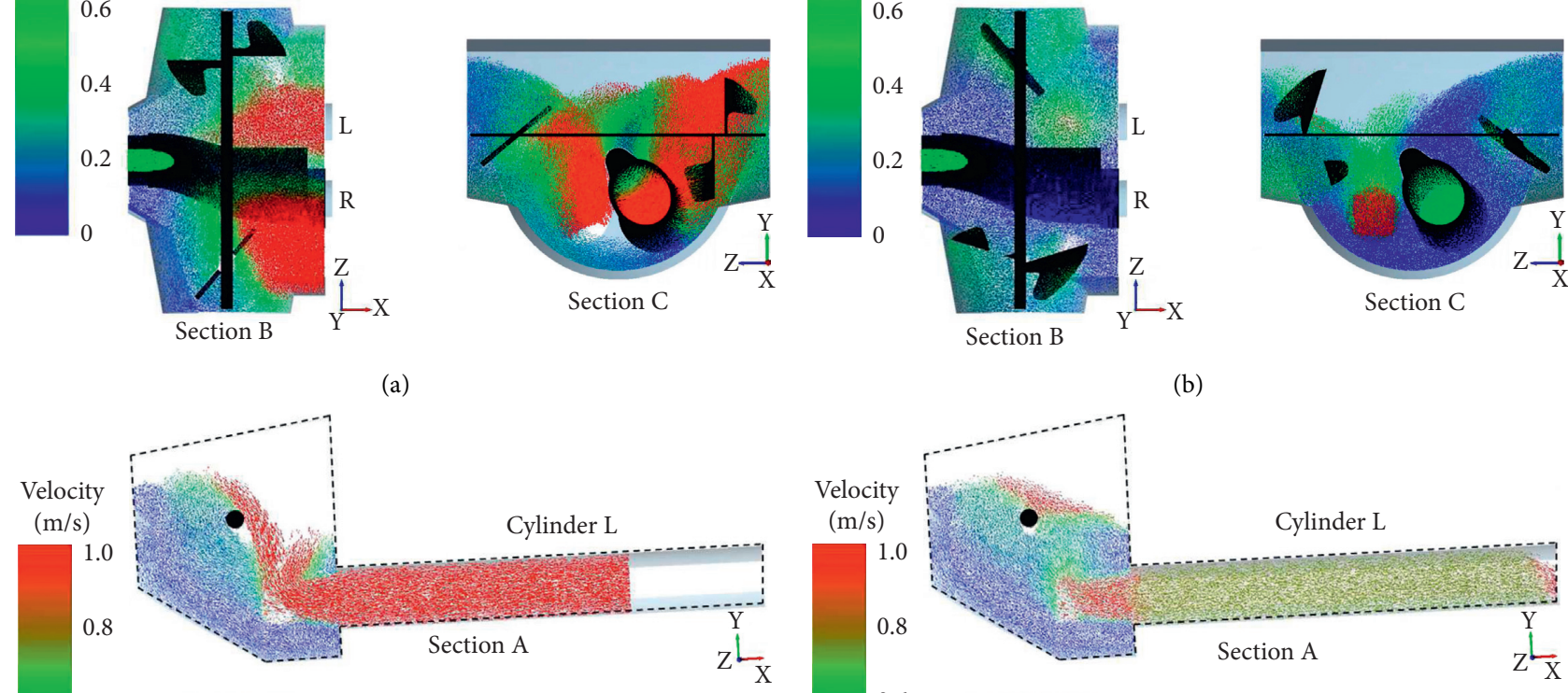

Section B

(b)

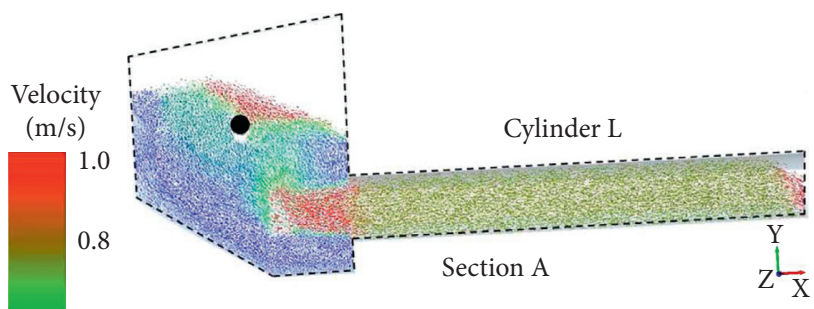

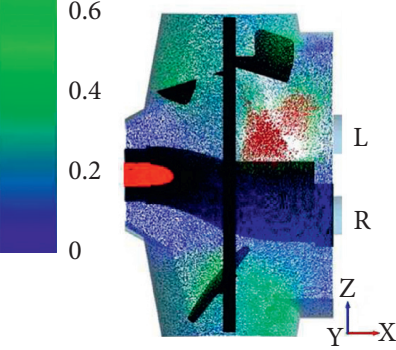

Section B

(c)
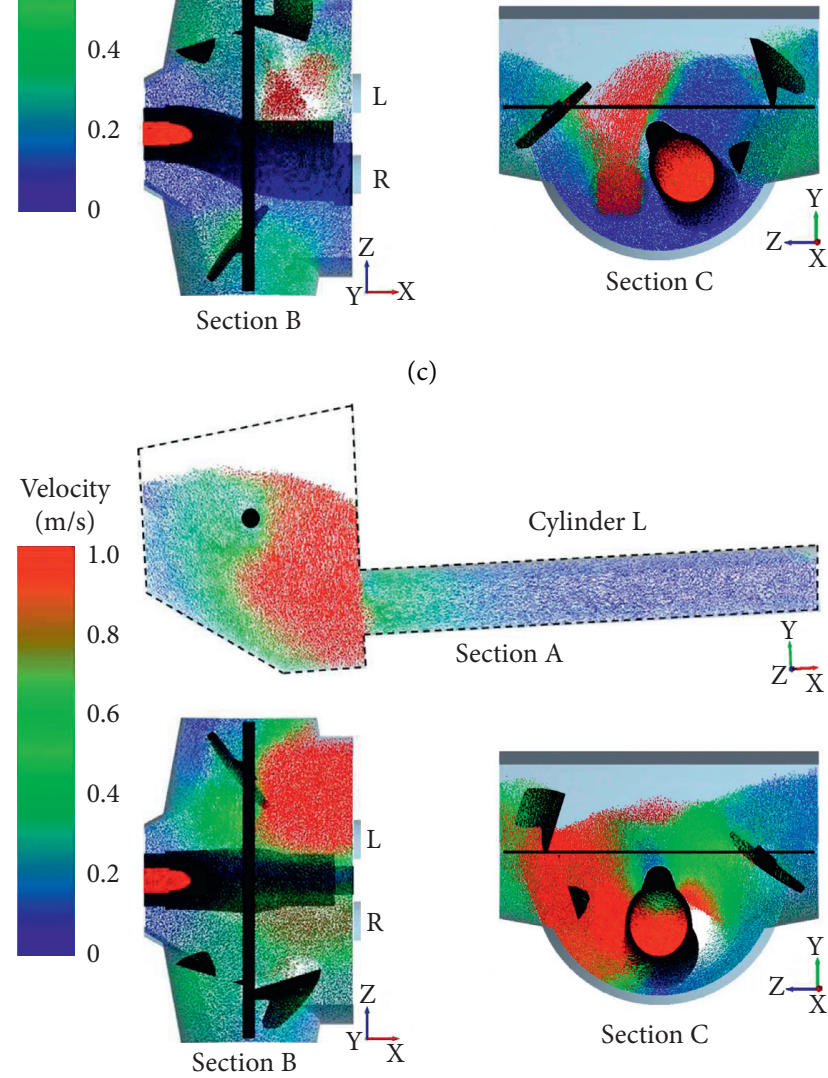

Section C

X

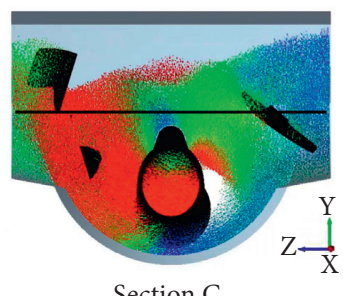

Cylinder L

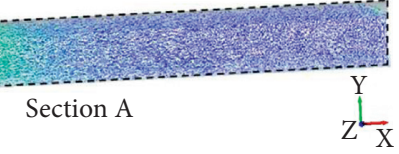

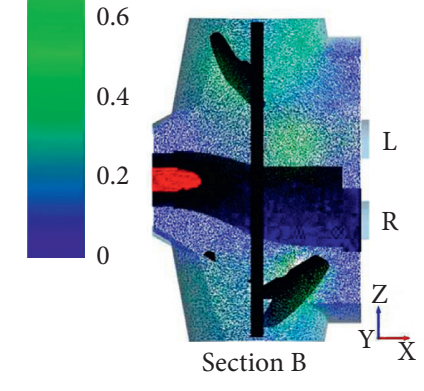

(d)

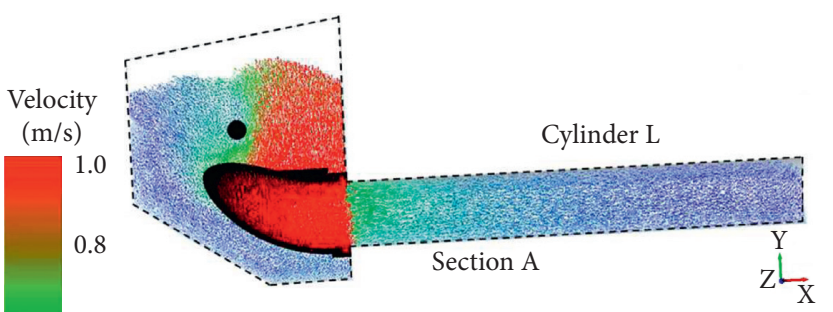

(e)
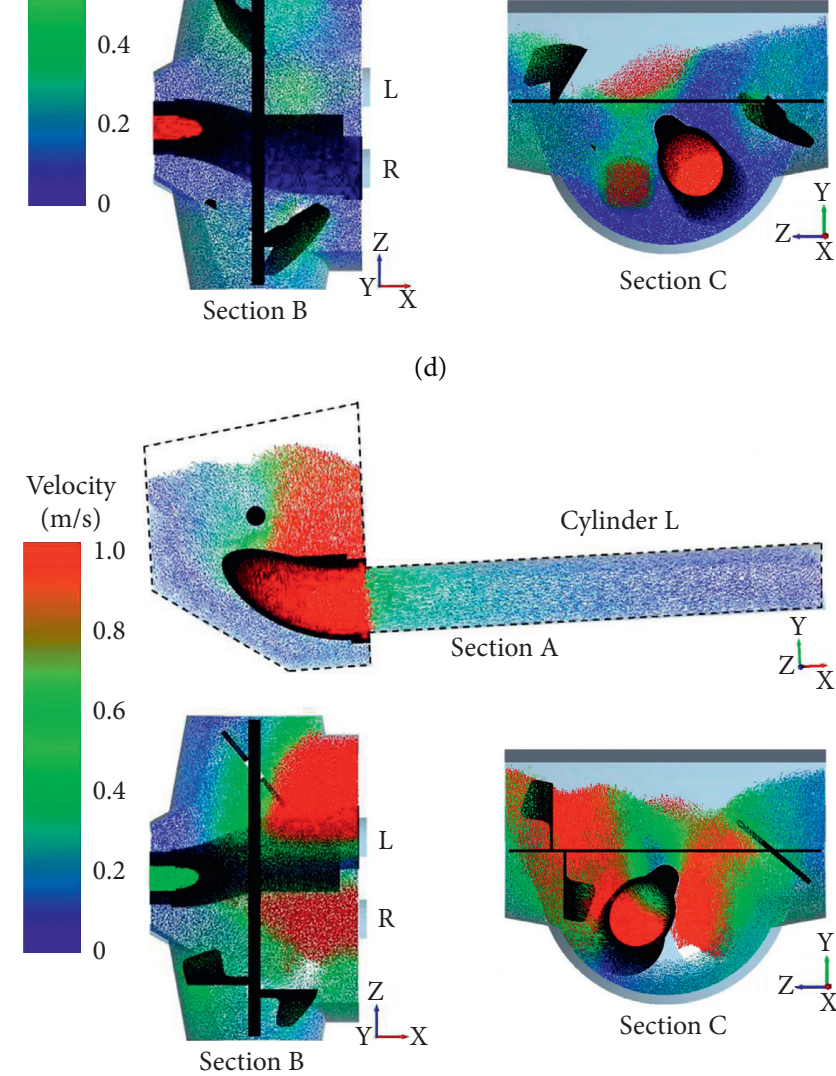

Section C

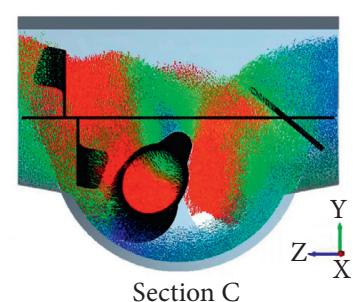

(f)

Figure 13: The concrete flow field of sections A, B, and C at different times: (a) $t=5.9 \mathrm{~s}$, (b) $t=6.4 \mathrm{~s}$, (c) $t=7 \mathrm{~s}$, (d) $t=7.5 \mathrm{~s}$, (e) $t=7.6 \mathrm{~s}$, and (f) $t=7.7 \mathrm{~s}$. 


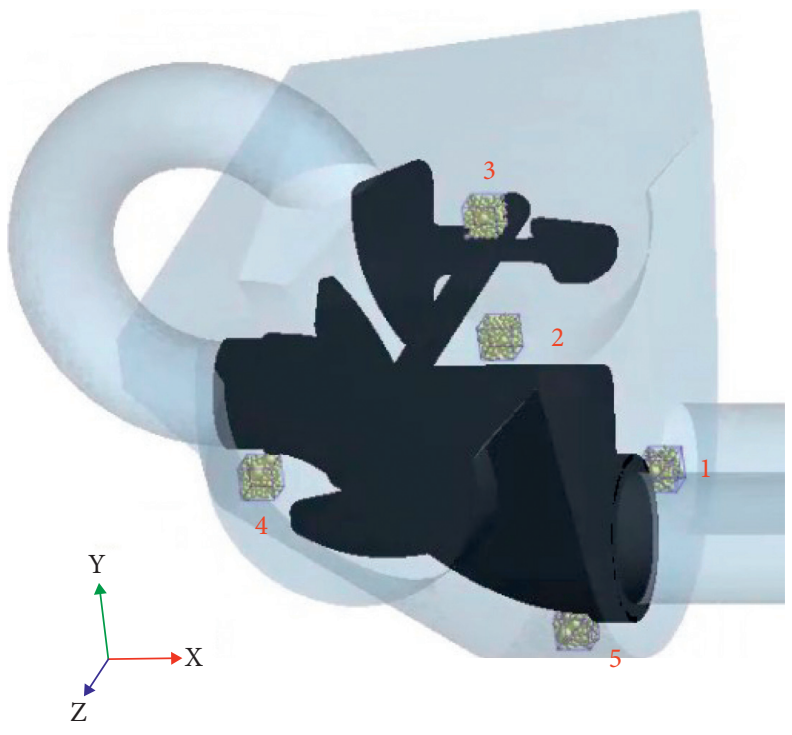

Figure 14: Five typical positions.

TABle 4: The center coordinates of grids.

\begin{tabular}{lccc}
\hline Position & $X$ & $Y$ & $Z$ \\
\hline 1 & 347.5 & -210 & -147.5 \\
2 & 0 & -70 & -400 \\
3 & 0 & 160 & -550 \\
4 & -337.5 & -160 & 0 \\
5 & 250 & -380 & 147.5 \\
\hline
\end{tabular}

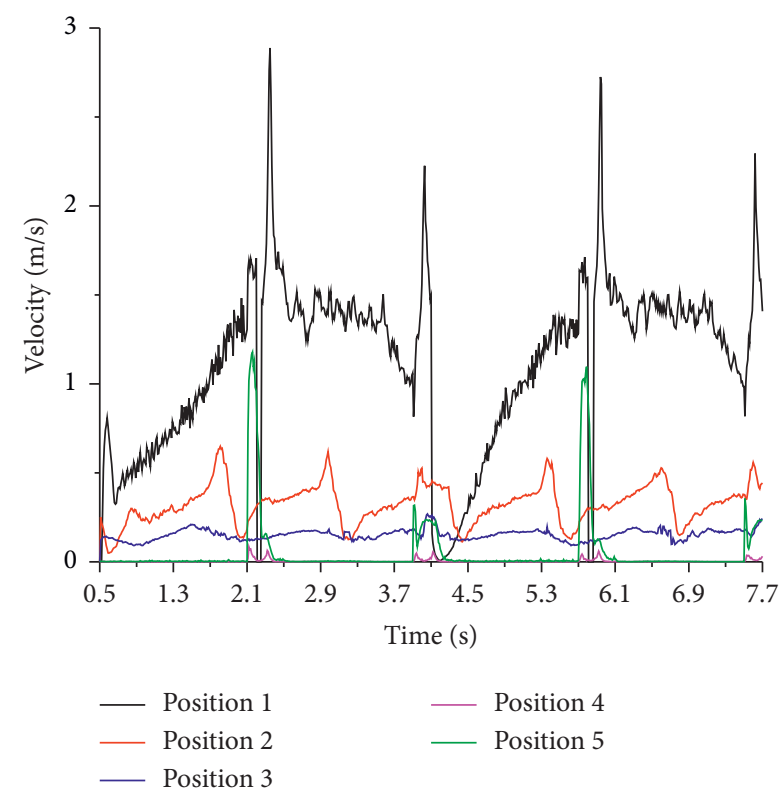

(a)

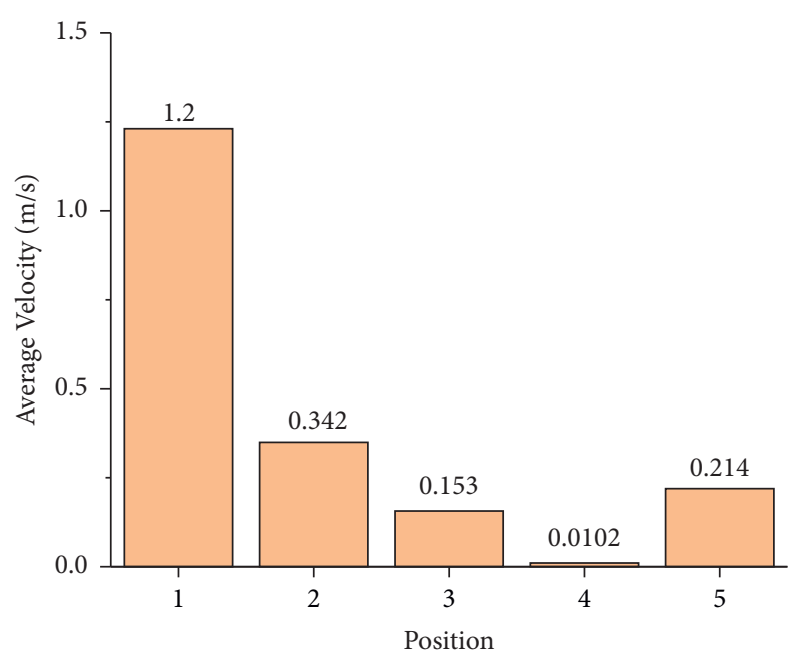

(b)

FIGURE 15: The change of particle velocity with time (a) and the average particle velocity at different locations (b).

is the same as the pumping cycle. The particle velocity fluctuates greatly during the reversing phase of the S-pipe $(2.1-2.3 \mathrm{~s}, 3.9-4.1 \mathrm{~s}, 5.7-5.9 \mathrm{~s}, 7.5-7.7 \mathrm{~s})$, including the maximum value. It is relatively stable in the early and middle stages and has a slow downward trend at the end of suction. It is shown that the particle flow velocity caused by the reversal of the S-pipe is greater than that caused by the suction. The change of particle velocity in position 2 is 
related to the rotation of the stirring blade, and the velocity always reaches the maximum when the stirring blade is swept. Position 3 particle velocity changes very little. The particles of position 4 and position 5 are driven only when the S-pipe is reversing, and the particle velocity can be ignored at other times. When the $S$ tube reverses, the particle velocity of position 4 is small while that of position 5 is large. During the whole pumping process, the average particle velocity of the 5 positions is $1,2,5,3,4$ in descending order.

In terms of the particle flow velocity, the particle flow caused by the reversing work of the S-pipe is the largest, followed by the particle flow caused by the suction work of the conveying cylinder, and the particle flow caused by the stirring work of the stirring blade is the smallest. The particle flow velocity near the hopper wall near the suction side is larger than that far from the suction side. The S-pipe swinging time is short, and the stirring blade has a greater auxiliary effect on the suction work in a long time.

Figure 16 is the flow trajectory of concrete particles near the stirring blade on the suction side. During the suction process, the concrete particles near the axis of the stirring blade form an obvious circulation under the continuous stirring of the stirring blade. The concrete particles near the suction port of the conveying cylinder and above the suction port of the piston cylinder in the hopper continuously flow into the conveying cylinder.

All in all, the stirring work makes the concrete particles in the hopper form an annular flow field around the stirring axis, ensuring the flow performance of the fresh concrete, and transporting concrete particles to the suction port of the conveying piston cylinder, thereby the suction of pumping system is promoted.

\section{The Influence of the Stirring Blade Parameters}

4.1. The Numerical Simulations. The stirring blade is composed of a stirring axis and four blades, which two blades are distributed at both ends of the axis. In the initial model of the stirring blade, the blade does not open holes, the angle between the blade plane and the axis (installation angle $\alpha$ ) is $40^{\circ}$, and the stirring blade rotates at a constant speed of $25 \mathrm{r} / \mathrm{min}$ (rotation speed $n$ ) when working, as shown in Figure 17. In the following, the blade will be opened with a hole, in which the edges distance $d$ refers to the distance between the inner and outer edges, as shown in Figure 18.

In this paper, the rotation speed $n$, the installation angle $\alpha$, and the edges distance $d$ of the stirring blade are considered as influencing factors that each factor is set to 4 levels. The suction efficiency $\eta$ of the pumping suction process and the stirring resistance torque $T_{M}$ of the stirring blade are considered as indicators to explore the influence on the suction process. The single-factor comparison scheme is designed, which included a total of 10 simulations, as shown in Table 5. Among them, only the rotation speed is changed in simulations 1, 2, 3, 4; only the installation angle is changed in simulations $2,5,6,7$; only the edges distance $d$ is changed in simulations $2,8,9,10$.

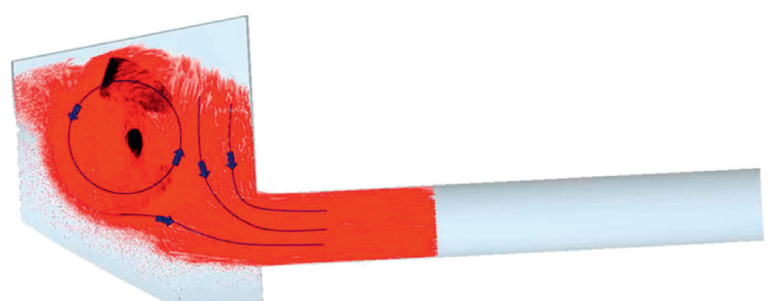

Figure 16: The flow trajectory of concrete. Particles near the stirring blade on the suction side.

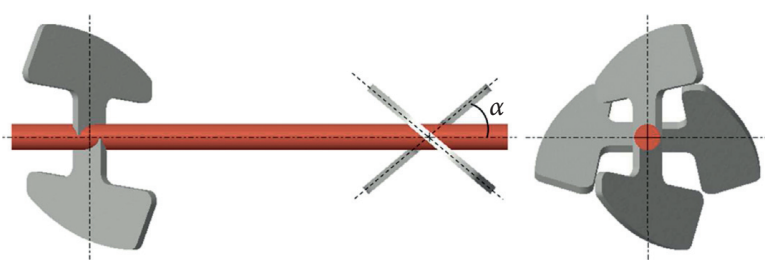

Figure 17: The initial model of the stirring blade.

4.2. Results of Simulations. Figure 19 is the variation of the stirring resistance torque with time from the results of simulations. The change of the resistance torque of the mixing blade $T_{M}$ is periodic and the period is $1.8 \mathrm{~s}$ which is the sum of the single suction time and the single S-pipe reversing time. The stirring resistance torque always fluctuates greatly during the S-pipe reversing period, which reaches the peak value at the beginning, and reaches the valley value at the end. The increase and decrease of the stirring resistance torque at different rotation speeds are occurred at the different time, while the trends of stirring resistance torque with different installation angles and different edges distances are the same.

First, the influence of the rotation speed of the stirring blade on pump suction is revealed by comparing the results of simulations 1, 2, 3, and 4, as shown in Figure 20(a). As the rotation speed increases, the suction efficiency $\eta$ increases and the average of stirring resistance torque $\overline{T_{M}}$ increases. Second, the influence of the installation angle of the stirring blade on pump suction is revealed by comparing the results of simulations 2, 5, 6, and 7, as shown in Figure 20(b). As the installation angle $a$ increases, the suction efficiency is almost unchanged and the average of stirring resistance torque decreases. Third, the influence of the edges distance $d$ of the stirring blade on pump suction is revealed by comparing the results of simulations $2,8,9$, and 10 , as shown in Figure 20(c). Compared with the base blade, the suction efficiencies of three perforated blades are slightly reduced, and they are almost the same. In addition, the average stirring resistance torque of the perforated blades is greatly reduced. As the edges distance decreases, the opening area increases, and the average of stirring resistance torque decreases.

The rotation speed has a great influence on the suction efficiency, while the installation angle and the edges distance have almost no influence on the suction efficiency. Moreover, with the increase of the rotation speed, installation 


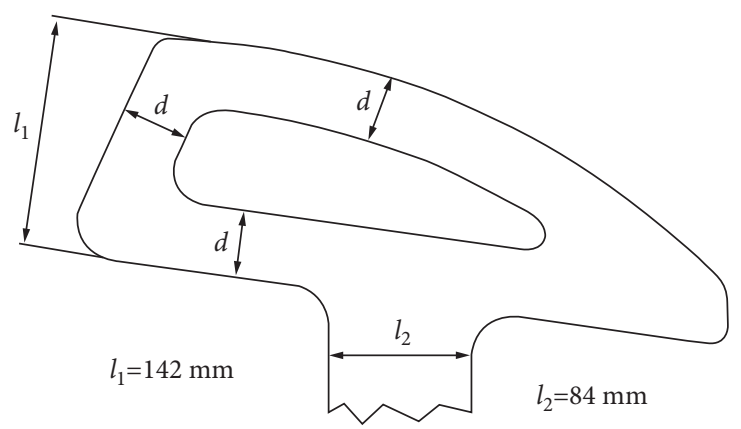

FIGURE 18: The blade with a hole.

TABle 5: The single-factor comparison scheme.

\begin{tabular}{lccc}
\hline No. & $\begin{array}{c}\text { Edge distance } d \\
(\mathrm{~mm})\end{array}$ & $\begin{array}{c}\text { Installation angle } \alpha \\
\left({ }^{\circ}\right)\end{array}$ & $\begin{array}{c}\text { Rotation speed } n \\
(\mathrm{r} / \mathrm{min})\end{array}$ \\
\hline 1 & - & 40 & 20 \\
2 & - & 40 & 25 \\
3 & - & 40 & 30 \\
4 & - & 40 & 35 \\
5 & - & 35 & 25 \\
6 & - & 45 & 25 \\
7 & - & 50 & 25 \\
8 & 50 & 40 & 25 \\
9 & 40 & 40 & 25 \\
10 & 30 & 40 & 25 \\
\hline
\end{tabular}

“-” means that the mixing blade does not perforate.

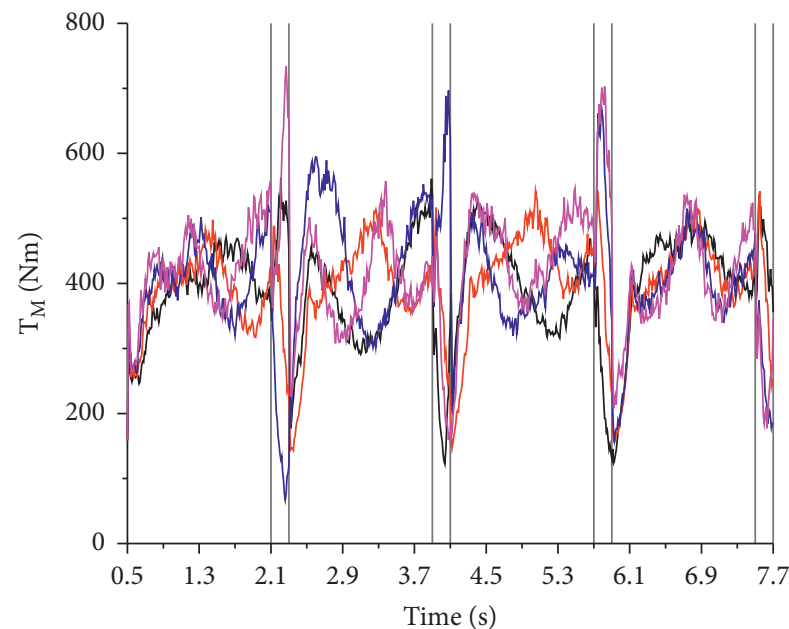

- Simulation 1 Simulation 2
- Simulation 3

— Simulation 4

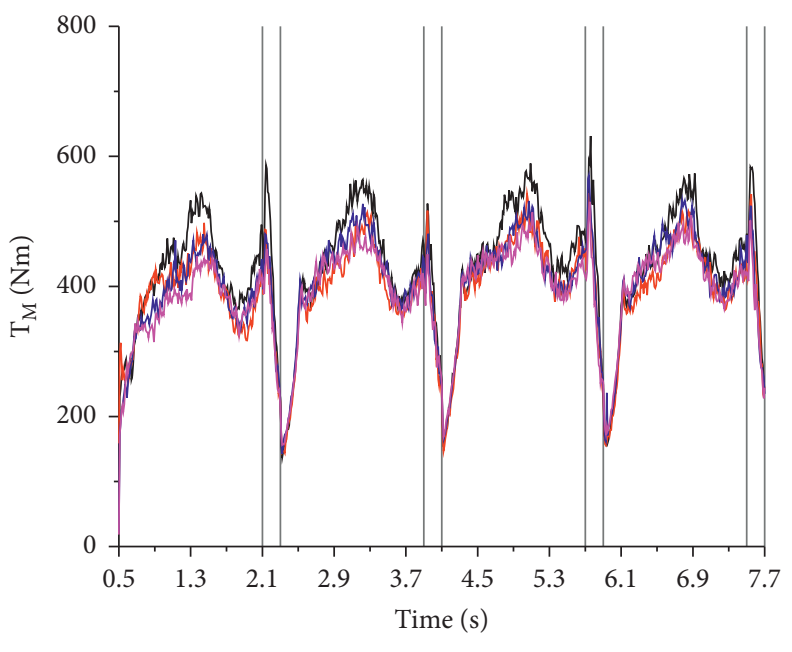

- Simulation 2

- Simulation 6

_ Simulation 7

(a)

(b)

Figure 19: Continued. 


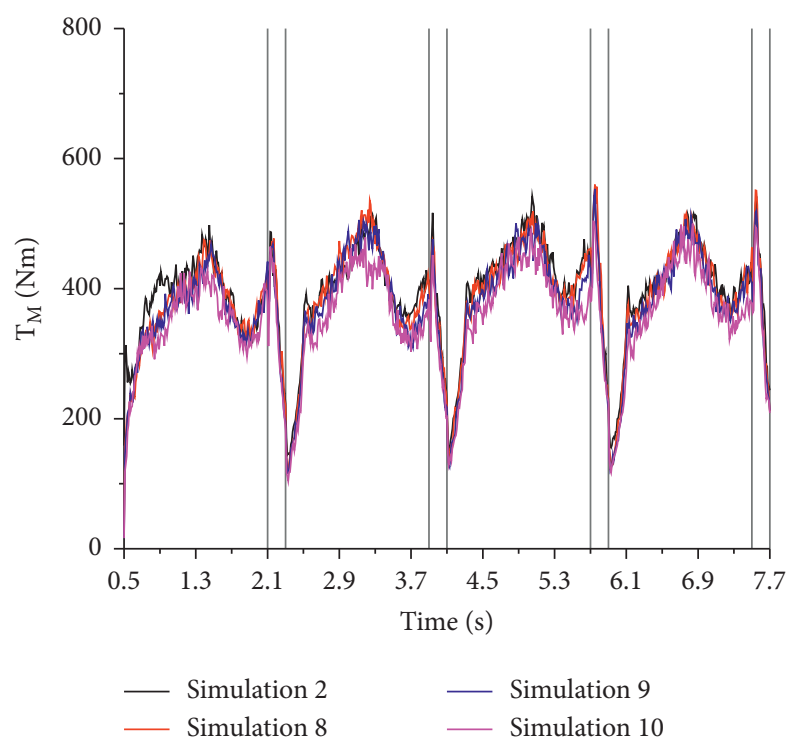

(c)

Figure 19: The variation of the stirring resistance torque with time.

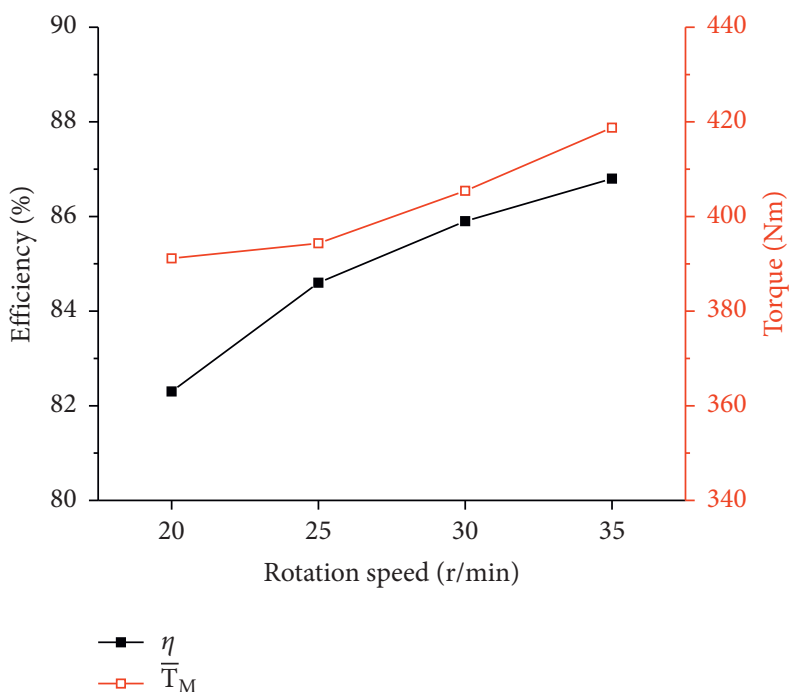

(a)

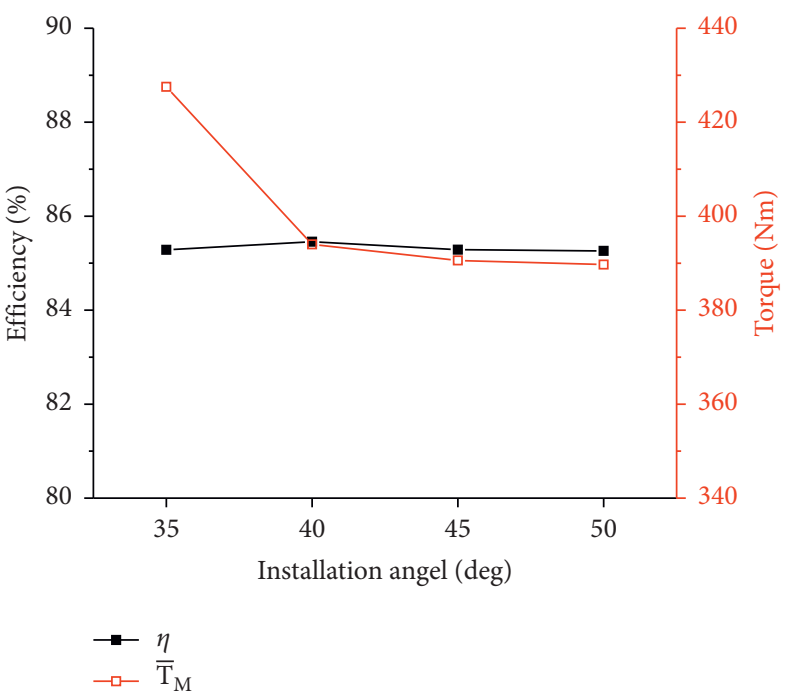

(b)

Figure 20: Continued. 


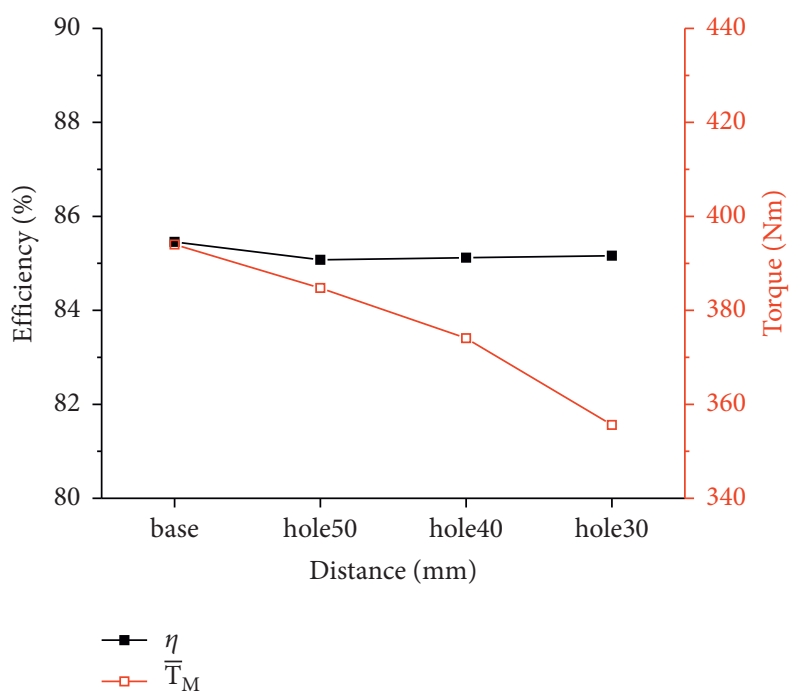

(c)

Figure 20: The influence of the rotation speed (a), installation angle (b), and edge distance (c) of the stirring blade on the pump suction.

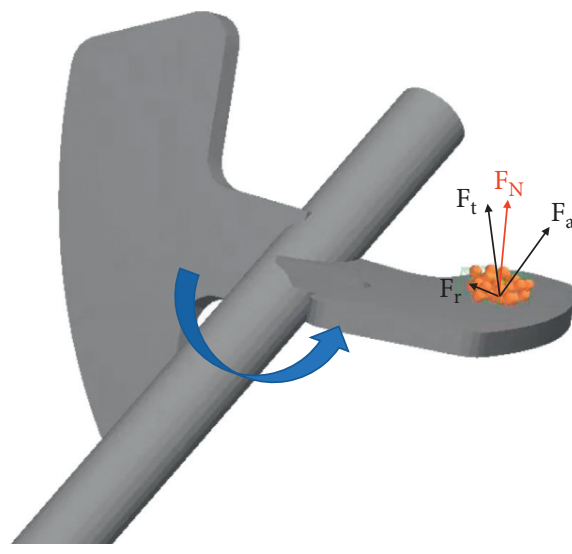

(a)

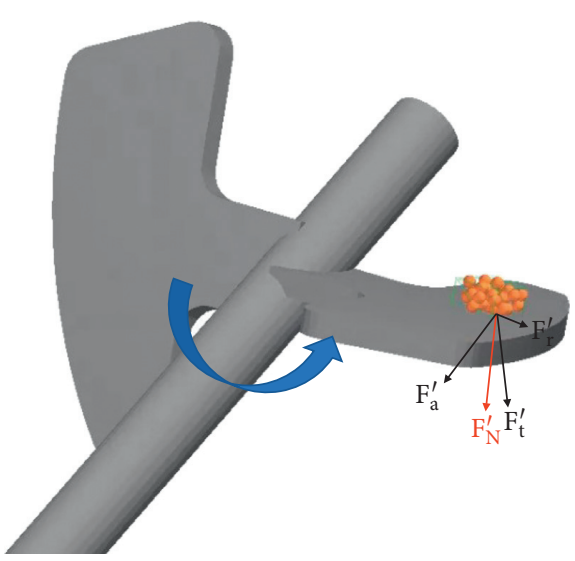

(b)

FIgURE 21: The force analysis of the concrete particles' unit (a) and the blade unit (b).

angle, and edges distance, the stirring resistance torque of the stirring blade increases.

\subsection{The Influence Mechanism of the Stirring Blade Parameters.} The interaction between the stirring blade and the concrete particles is mainly generated the point where they directly collide with each other. Concrete particles in direct collision with stirring blades are divided into many particle units. Through the force analysis of any one of the concrete particle units, this unit is mainly driven by the normal force $F_{N}$ of the blade. Correspondingly, the stirring blade unit is mainly subjected to the resisting force $F_{N}$ ' of concrete particles, which is opposite to the normal force $F_{N}$ of the blade, as shown in Figure 21. The resisting force $F_{N}$ ' is decomposed into the radial force $F_{r}^{\prime}$ pointing to the stirring axis, the axial force $F_{a}^{\prime}$ parallel to the stirring axis, and the tangential force $F_{t}^{\prime}$ tangent to the rotation direction of the stirring axis. The relationship between tangential force $F_{t}^{\prime}$ and resisting force $F_{N}^{\prime}$ is shown in formula (7). The radial force and axial force are parallel to the rotation direction of the stirring blade, which does not hinder the rotation of the stirring blade. The stirring resistance torque is mainly the synthesis of the torque of the tangential force of each stirring blade unit to the stirring blade axis. Therefore, the stirring resistance torque mainly comes from the tangential force.

$$
F_{t}^{\prime}=F_{N}^{\prime} \cos \alpha
$$

Changing the rotation speed is mainly to change the resisting force. With the increase of rotation speed, the resisting force increases, so the stirring resistance torque increases. The installation angle is changed, and the tangential force changes accordingly, as shown in formula (7). The larger the installation angle $\alpha$, the smaller the tangential force and the smaller the stirring resistance torque. When 


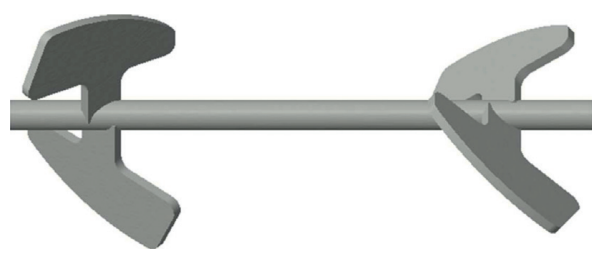

(a)

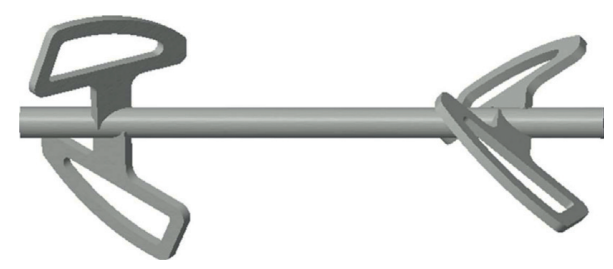

(b)

FIgURE 22: The (a) initial stirring blade and (b) optimized stirring blade.

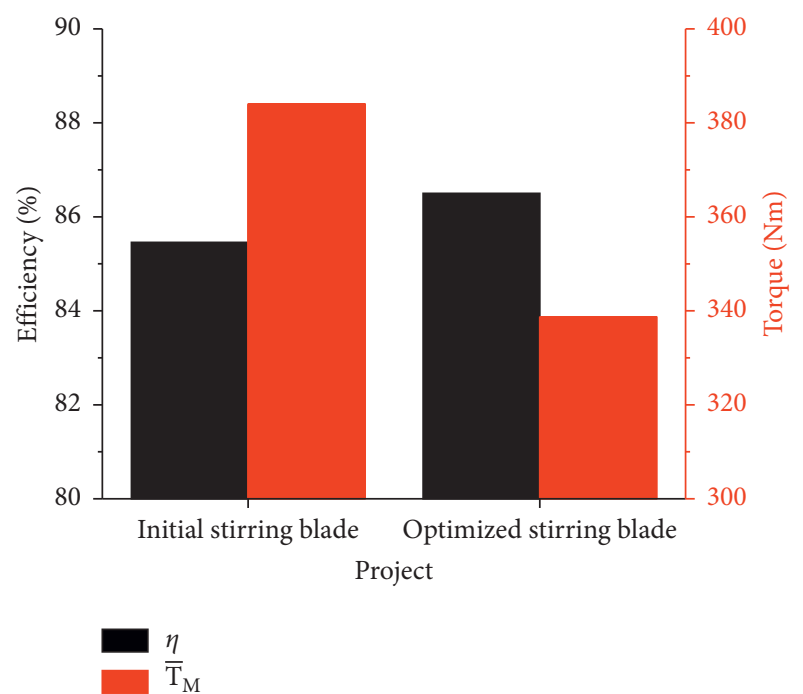

(a)

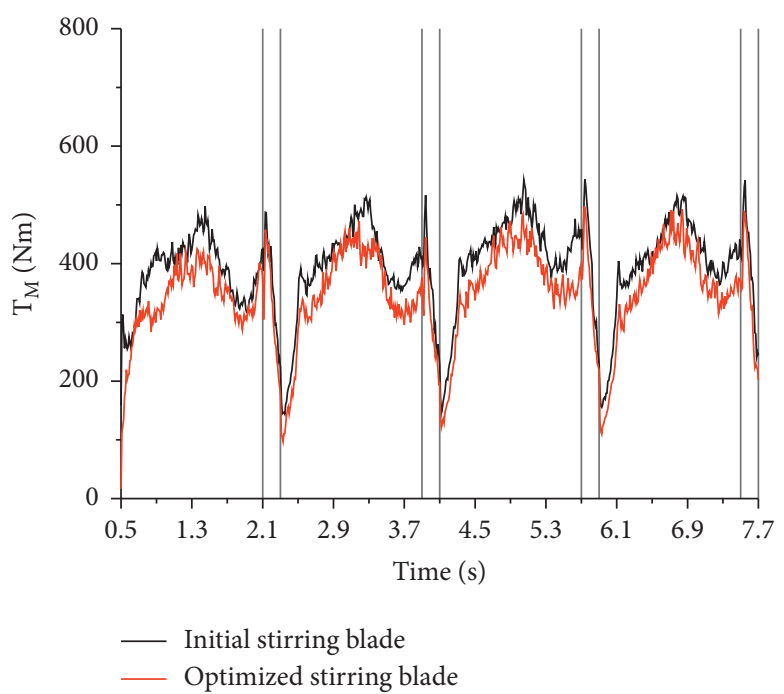

(b)

FIgURE 23: The simulation results of the initial stirring blade and the optimized stirring blade.

the stirring blade is opened a hole, the area of the stirring blade is changed with the edges distance $d$. With the decrease of the edges distance, the area of the blade decreases, and the units of the stirring blade decreases, so the stirring resistance torque decreases. These rules are consistent with the simulation results.

\section{Optimization}

In order to reduce the stirring energy consumption of the stirring blade without reducing the suction efficiency, the structure of the initial stirring blade is optimized. The installation angle is improved from $40^{\circ}$ to $50^{\circ}$ and the blade is opened with a hole which the edges distance is $30 \mathrm{~mm}$, as shown in Figure 22.

The pumping suction with the optimized stirring blade is simulated compared with the initial stirring blade. The simulation results are shown in Figure 23. After optimization, the trend of the stirring resistance torque is unchanged, the suction efficiency is increased by $1.22 \%$, and the stirring resistance torque of the mixing blade is reduced by $11.8 \%$. It is consistent with the expectation which reduces the stirring energy consumption of the stirring blade without reducing the suction efficiency.

\section{Conclusions}

The DEM model of fresh concrete was established. The gap between experiments and simulations results of slump flow tests and L-box tests was less than $5 \%$, so the feasibility of simulating the flow behavior and rheological properties of the fresh concrete was proved.

The geometric structure of pumping system was simplified. The suction force of the conveying cylinder was customized by the API function of DEM to realize the suction work. The difference of suction efficiency in experiments and simulations was less than $0.1 \%$. Therefore, the numerical simulation of the suction process was completed and feasible.

The concrete particles flow during the suction process was further studied. In terms of the particle flow velocity, the particle flow caused by the reversing work of the S-pipe was the largest, followed by the particle flow caused by the suction work of the conveying cylinder, and the particle flow caused by the stirring work of the stirring blade was the smallest. The stirring blade maked the concrete particles in the hopper form an annular flow field around the stirring axis, so the suction of pumping system was promoted.

In addition, the influence of the stirring blade was explored. The rotation speed had a great influence on the 
suction efficiency, while the installation angle and the edges distance had almost no influence on the suction efficiency. Moreover, with the increase of the rotation speed, installation angle, and edges distance, the stirring resistance torque of the stirring blade increased.

An optimized stirring blade was established and compared with the initial stirring blade. After optimization, the suction efficiency was increased by $1.22 \%$, and the stirring resistance torque of the mixing blade was reduced by $11.8 \%$. The stirring energy consumption of the stirring blade was reduced without the suction efficiency reduced.

\section{Data Availability}

The data used to support the findings of this study are available from the corresponding author upon request.

\section{Conflicts of Interest}

The authors declare that they have no conflicts of interest.

\section{Acknowledgments}

This work was supported by the Natural Science Foundation of Hunan Province (2021JJ20009, 2020JJ5541, 2020JJ5538, 2019GK4025, and 2019JJ20015) and the NSFC projects of China (52075465 and 11772135). The authors are grateful for the financial support for this research.

\section{References}

[1] Y. Wu, W. Li, and Y. Liu, "Fatigue life prediction for boom structure of concrete pump truck," Engineering Failure Analysis, vol. 60, pp. 176-187, 2016.

[2] S.-J. Lee, I.-S. Chung, and S.-Y. Bae, "Structural design and analysis of CFRP boom for concrete pump truck," Modern Physics Letters B, vol. 33, no. 14n15, Article ID 1940033, 2019.

[3] A. L. Wang, Z. Q. Shi, C. X. Yuan, and Y. Q. Hu, "Optimal design of concrete pumping displacement control," Advanced Materials Research, vol. 1586, pp. 238-242, 2012.

[4] N. Roussel, M. R. Geiker, F. Dufour, L. N. Thrane, and P. Szabo, "Computational modeling of concrete flow: general overview," Cement and Concrete Research, vol. 37, no. 9, pp. 1298-1307, 2007.

[5] M. Cremonesi, L. Ferrara, A. Frangi, and U. Perego, "Simulation of the flow of fresh cement suspensions by a Lagrangian finite element approach," Journal of Non-newtonian Fluid Mechanics, vol. 165, no. 23, pp. 1555-1563, 2010.

[6] S. Tichko, J. Van De Maele, N. Vanmassenhove et al., "Numerical simulation of formwork pressure while pumping selfcompacting concrete bottom-up," Engineering Structures, vol. 70, pp. 218-233, 2014.

[7] E. Secrieru, W. Mohamed, S. Fataei, and V. Mechtcherine, "Assessment and prediction of concrete flow and pumping pressure in pipeline," Cement and Concrete Composites, vol. 107, Article ID 103495, 2020.

[8] H. Hoornahad and E. A. B. Koenders, "Simulating macroscopic behavior of self-compacting mixtures with DEM," Cement and Concrete Composites, vol. 54, pp. 80-88, 2014.

[9] V. Mechtcherine and S. Shyshko, "Simulating the behaviour of fresh concrete with the Distinct Element Method - d," Cement and Concrete Composites, vol. 55, pp. 81-90, 2015.
[10] H. Ji, J. Caiyun, L. Yue et al., "Simulation of motion behavior of concrete in pump pipe by DEM," Advances in Civil Engineering, vol. 2021, Article ID 3750589, 16 pages, 2021.

[11] G. Lu, K. Wang, and T. J. Rudolphi, "Modeling rheological behavior of highly flowable mortar using concepts of particle and fluid mechanics," Cement and Concrete Composites, vol. 30, no. 1, pp. 1-12, 2007.

[12] Y. Tan, H. Zhang, D. Yang, S. Jiang, J. Song, and Y. Sheng, "Numerical simulation of concrete pumping process and investigation of wear mechanism of the piping wall," Tribology International, vol. 46, no. 1, pp. 137-144, 2011.

[13] M. S. Choi, Y. J. Kim, and J. K. Kim, "Prediction of concrete pumping using various rheological models," International Journal of Concrete Structures and Materials, vol. 8, no. 4, pp. 269-278, 2014.

[14] M. Choi, N. Roussel, Y. Kim, and J. Kim, "Lubrication layer properties during concrete pumping," Cement and Concrete Research, vol. 45, pp. 69-78, 2013.

[15] Y. Zhan, J. Gong, Y. Huang, C. Shi, Z. Zuo, and Y. Chen, "Numerical study on concrete pumping behavior via local flow simulation with discrete element method," Materials, vol. 12, no. 9, 2019.

[16] J. Shengqiang, C. Xiaodong, C. Guodong et al., "Optimization of fresh concrete pumping pressure loss with CFD-DEM approach," Construction and Building Materials, vol. 276, 2021.

[17] M. Liu, L. Tan, and S. Cao, "Theoretical model of energy performance prediction and BEP determination for centrifugal pump as turbine," Energy, vol. 172, 2019.

[18] M. Liu, L. Tan, Y. Xu, and S. Cao, "Optimization design method of multi-stage multiphase pump based on Oseen vortex," Journal of Petroleum Science and Engineering, vol. 184, no. C, 2020.

[19] X. Wenyang and T. Lei, "Design method of controllable velocity moment and optimization of pressure fluctuation suppression for a multiphase pump," Ocean Engineering, vol. 220, 2020 (prepublish).

[20] M. Liu, L. Tan, and S. Cao, "Influence of geometry of inlet guide vanes on pressure fluctuations of a centrifugal pump," Journal of Fluids Engineering, vol. 140, 2018.

[21] K. L. Johnson, K. Kendall, and A. D. Roberts, "Surface energy and the contact of elastic solids," Proceedings of the Royal Society of London - Series A: Mathematical and Physical Sciences, vol. 324, no. 1558, pp. 301-313, 1971. 